\title{
DETERMINAÇÃO DA BASICIDADE RELATIVA DAS AMINAS POR RESSONÂNCIA CICLOTRÔNICA DE IONS
}

\author{
Tese apresentada à \\ Comissão Julgadora do Concurso à \\ Docência-Livre de Físico-Química do \\ Departamento de Química Fundamental do \\ Instituto de Química da \\ Universidade de São Paulo.
}

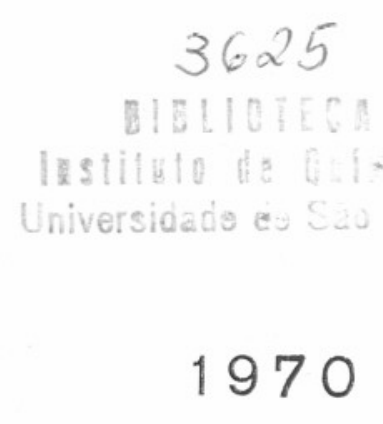




\section{AGRADECIMNNTOS}

Ao Conselho Nacionol de Pesquises pelo concasgão de uno bôlsa de viagen poṛe estagiar ne Universidede de Stonfora, e pela generosa doç̧̃̃o do espectrônetro de ressonêncic ciclotrônica.

Lo Professor John D. Baldeschwieler por tor oforecido a oportunidade de estagiar en seu laboratorio, o pelo seu entusicsmo na pesquise. Lo grupo de pesquise do stonford, e espocialmonto a Iarry Blair, por tor no ajudado o sugerido o tipo de probleme desenvolvido nêsto trabolho.

Aos que pocientemonte corregirem o porte mois difícil de tese para mim: escrevel-la em portugues. Dosteco nêste aspecto - grande colaborcção de Ione Polacow, Dr. Divo I. Sanjoto o Fernondo Galembeck. Os crros que nindo persistem dovem sor atribuidos exclusivonente so autor, o es suas dificuldades com a Ingua portuguesa o a méquina de escrever.

Los Professoros Simño Mathics o Paschoal Soniso polo ostinulo constanto nas minhes atividedos universitérins no Brasil. 


\section{INDICE}

Introdução

Capitulo I: Caratorísticos do movinonto de um fon nun ormpo magnético。

I.I Movinonto de un ín num compo mognético

I.2 Equaçõo de un lon na presença do w compo megnetico unifomo e un canpo eletrico olternonto - -

I.3 Aplicnç̃o químico dos principios do ciclotron …...... 9 Copítulo II Espectroscopie de rossonêncin ciclotrônice de íns

II.] Rosumo histórico

II.2 Lspectrômetro -

II.3 Considernções próticas sôbro o movimonto dos fons no colc do espectrômetro -

I.4 Efeito de ressonência dupla c coolononto dos movimen... tos entre íons _- 24

Copítulo III: Estudo do basicidade relotive dos aninos

III.I Introduç̃̃o - 26

III.2 Corotoristice gorel dos espectros de RCI dos cninos - 27

III.3 Considerçõos sôbro as reçõos entre íns o noléculcs ne fase gososa -

III.4 Significado cinótico de cxporiência de rossonência ciclotrônica - 36

III.5 Uso de varieção da constante de volocidade com onorgí tronslacional do lon recgento pare deteminor a afinidade protônice des cminss -

III.6 ISpuctros e resultrdos - 43

II.7 Andise cretico da ordon de bosicidede por ressonêncie duple -

III.8 Escola de bosicidade relativ das oninas .................. 55

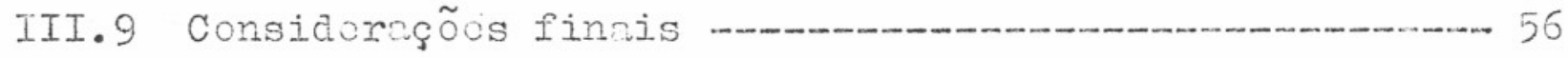

Resuno -

Bibliograitic 
o desenvolvimento recente de uma nova técnica espectroscopica, chameda ressonincia ciclotrônica de fons (RCI), abre caminho para uma serrie de experiências que poderiam ser cetalogadas de espectroscopia quínica. Bste último nome 6 muito apropriado para o caso da RCI, porcue seu volor principal consiste justemente na capacidade de estudar reacões quinicas e processos dinemicos de lons na fase gasosa por um método espectroscbpico. Assim, em muitas aplicações, as "transições espectroscopicas" correspondem às reações de um fon de massa. II para produzir um outro lon de massa I'. $^{\prime}$

Este trabalho usa os princfpios da RCI para estudar certo tipo de reações des amines protonadas na fose cesosa. Os resultados, associados com certas caraterísticas das reacões entre Lons e moléculas na fase gesosa, pemitem estebelecer a bosicidade intrinseca relativa das aninas alifétices, independente de qualquer efejto de solvatação. Ista escola de basicidade é de interêsse fundamental em problemas cuinicos, e na compreensão da relação entre estrutura e comportanento quimico.

Como as conclusões desta tese dependem dos principios bésicos da RCI, Grande destaque é dado nos primeiros nanítulos aos principio flsicos e experimentais do método. Isto talvez consige dar uma ideia global da RCI, sua aplicasão a problema indicaco, e outras possiveis cplicacões. 
I. I Movimento de un fon num canpo mame tico ${ }^{1,2}$

A trajetoric no vécuo de uma partícula com carga elétrice I no presença de un campo magnético, if é representeda pela equoção (I-I), (em unidades gaussianas)

$$
\vec{F}=\frac{q}{6}(\vec{c} \times \vec{H})
$$

Nesta equação, as grandezas ffisicas de interêsse são definidas da seguinte maneira:

F-- fôrça que atua na particula devido ao campo magnetico

v -. velocidade da particula

c -.- velocidade do Iuz.

Se o compo magnetìco fôr uniforme, a trajetoria da partfcula sert una orbita circular, num plano perpendicular a. campo magnetico. Entretcrito, a componente do movimento original da port1culo na direção paralela a II não será alterade por esta fôrça.

O movimento circular de un Lon nesta situação estaré caraterizcdo por un certo raio, e pela frequência, ou periodo, do movimento. A Irequência e geralmente denominada frequência ciclotrônica, devido do uso desta frandeza bésica en Ifsica nuclear como clemento do ciclotron. As duas grandezas mencionadas podem ser obtidas facilmente a portir da relação de equilfbrio entare a ficticia fôrça contrifuga e a fôrça magnética.

$$
\frac{q}{c} v_{i} H=\frac{m v_{1}^{2}}{r}
$$


$\left(v_{i},-\right.$ componente de velocidado no plano perpendiculer d H). Dar

$$
r=\frac{m c v_{1}}{q H}
$$

c

$$
\omega_{c}=\frac{w_{i}}{r}=\frac{q H}{m c}
$$

Estas equações mostron que a frequência ciclotrônice, c por tanto o tempo necessério pare porcorrer una brbite, independer de velocidade do fon, mas dependen da massa do mesmo. hssim, pora un conjunto de Lons de massa m, haveró una distrịuição de brbitas de acôrdo com oq. (1-3), mas a frequência sork a mosna para todos os Lons. Estas considerações podom ser ilustradas numericamento para um fon de argônio num compo negnetico de 4.000 geuss, considerando une velocidede correspondento 2 volocidnde modia de $\mathrm{Ar}^{+}$d tomporotura anbionto. Pern ôste coso,

$$
\begin{gathered}
\omega_{c} / 2 \pi-153,6 \mathrm{kHz} \\
r-0,02 \mathrm{~cm} .
\end{gathered}
$$

I.2 Equecão de um fon na presenca de um conpo nognético uniforme a un canpe clétrico olternante

A trajotoria do ion considerada no ften I.I sofro dtorasõos quando un conpo elétrico altomente do froquêncic $w_{1}$ \& adicionado so probloma. O caso a ser detclhado nosta prrte e somelhante 20 movimonto de partículas num ciclotron: o campo olstrico 8 cplicado numa direção perpendicular no campo magnético. 
A equação goral do movimento de un Lon isoledo, neste caso, serk ropresentcde por eq. (1-5),

$$
\vec{F}=m \frac{d \vec{v}}{d t}=q \vec{E}(t)+\frac{q}{c}(\vec{v} \times \vec{H})
$$

Se a direção do compo megnético es definida ne direç̃̃o do eixo positivo $z$ de um sistema de coordenadas cortosionas, enquanto o carapo eletrico alternante oscilo sobre o dixo 프, es grandezas vetoriais podem ser definidas de acôrdo â Figura 1-1.

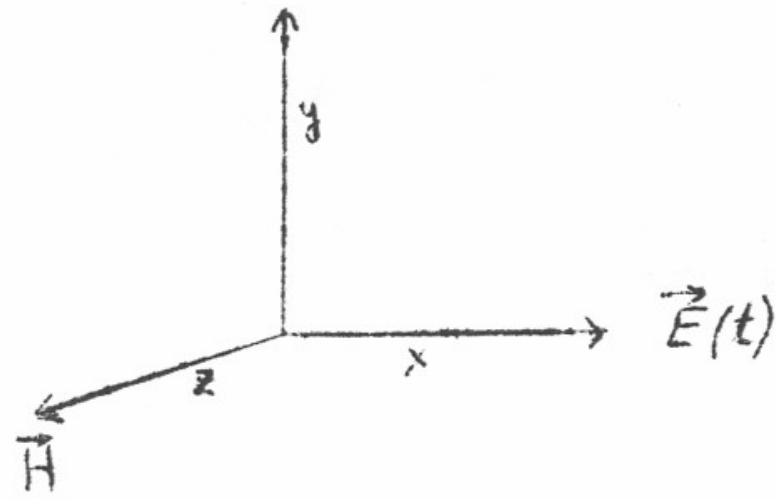

Figura 1-1

$$
\vec{H}=H \vec{k}, \quad \vec{E}(t)=E_{0} \operatorname{sen} \omega_{1} t \vec{i}, \omega_{6}=\frac{q H}{m c}
$$

- (Aqui, $\vec{i}, \vec{j}$, o $\overrightarrow{\underline{k}}$ representan como de costume vetores unitários nas direções $\underline{x}, \underline{y}, e \underline{z}$ )

A solução da equação diferencial (1-5), coreterizada explleitemente pelas eqs. (1-6) ganho en sentido físico se - compo elétrico altemante é decomposto vetoriclnonte om 2 campos elétricos girando em direção oposte, da maneira ilustrada pelas equeções $(1-7)$

$$
\vec{E}(t)=\overrightarrow{\mathrm{B}}_{1}(t)+\vec{E}_{2}(t)
$$




$$
\begin{aligned}
& \vec{E}_{1}(t)=\frac{d_{2}}{2} \quad E_{0}\left(\operatorname{sen} \omega_{1} t \vec{i}+\cos \omega_{1} t \vec{j}\right) \\
& \vec{E}_{2}(t)=\frac{1}{2} \quad E_{0}\left(\operatorname{sen} \omega_{z} t \vec{i}-\cos \omega_{1} t \vec{j}\right)
\end{aligned}
$$

A vantagem de exprimir o compo elétrico desta maneira é que o movimento circular explicado prèviamente, so e perturbado pelo campo eletrico quando $\boldsymbol{w}_{2} * w_{k}$. Isto equivale a dizer que na solução do problema é necessário considerar só aquela componente do compo eletrico que esto girando na mesma direção que o Ion.

Se $\mathrm{E}_{l}(t)$ es escolhido arbitrariamente como a componente de interêsse, a equação diferencial (1-5) pode ser desdobrada em três equações correspondentes às componentes do vetor velocidede.

$$
\begin{aligned}
& \frac{d v_{x}}{d t}=\frac{q}{2 m} E_{0} \operatorname{sen} \omega_{1} t+v_{y} w_{c} \\
& \frac{d v_{y}}{d t}=\frac{q}{2 m} E_{0} \cos \omega_{1} t-v_{x} w_{c} \\
& \frac{d v_{z}}{d t}=0
\end{aligned}
$$

As duas primeiras equações são equações diferenciais acoplades. Sua resolução pode ser obtida điferenciando a prineira equação com relação no tempo,

$$
\frac{d^{2} v_{x}}{d t^{2}}=\frac{\omega_{1} q}{2 m} F_{0} \cos \omega_{1} t+w_{c} \frac{d v_{y}}{d t}
$$

e substituindo $d v_{\mathrm{y}} / d t$ por seu valor ne equação (1-8). Assim, obtém-se,

$$
\frac{d^{2} v_{x}}{d t^{2}}+w_{t}^{2} v_{x}=\frac{E_{0}}{2 m}\left(w_{1}+w_{t}\right) \cos \omega_{1} t
$$


Resolvondo esta equaçño äiforencial, e tendendo ̀̀s condições de contômo se chega à solução pera $v_{X}(t)$.

$$
v_{x}(t)=\frac{q E_{0}^{*}}{2 m\left(\omega_{c}-\omega_{1}\right)}\left(\cos \omega_{1} t-\cos \omega_{c} t\right)+v_{1}^{0} \operatorname{sen}\left(\omega_{c} t+r\right)
$$

A solução para $\mathrm{v}_{\mathrm{y}}(\mathrm{t})$ pode ser obtida de naneira onáloga.

$$
v_{y}(t)=-\frac{q E_{0}}{2 m\left(\omega_{x}-\omega_{y}\right)}\left(\operatorname{sen} \omega_{c} t-\operatorname{sen} \omega_{c} t\right)+v_{1}^{2} \cos \left(\omega_{c} t+y\right)
$$

Aqui, $v_{l}^{0}$ se refere a velocidade no plano perpendiculer a H no tempo $t=0$, onquanto que $\gamma$ esimplesmente a fose inicial deste grandeze.

A soluçẽo rigorosa do problema, ou seja considerando $\mathbb{E}_{1}(t)$ e $\mathbb{E}_{2}(t)$, eficilmente obtida quendo se observe que $\mathrm{E}_{2}(t)$ corresponde 0.0 compo eletrico $\mathrm{E}_{1}(t)$ com errequencia $\pi-\omega_{1}$. DaI, a soluç̃o completa scria da forma.

$v_{x}(t)=\frac{q E_{0}}{2 m}\left(\cos \omega_{1} t-\cos \omega_{c} t\right)\left[\frac{1}{\omega_{c}-\omega_{1}}+\frac{1}{w_{c}+\omega_{1}}\right]+v_{L}^{\circ} \operatorname{sen}\left(\omega_{c} t+\gamma\right)$

e ume expressĩo semelhente pare $v_{\mathrm{y}}(t)$. Como na maior porto das experiências a serem considoredas $w_{1} \sim w_{t}$, o scgundo têrmo dentro do colchete pode ser desprezedo de acôrdo com a hipotese inicial.

As soluções ate aqui obtidas, e representadas pelas equações (I-II), (I-12) e (I-13), admiten que $w_{1} \neq w_{c}$. Nostes condições, o efeito do compo elétrico representa uma perturbeção no novinento circuler original, ou or outros têmos, - compo elétrico altemente modula o movimento circular con una frequência $\omega_{1}$. 
Quando a frequencia do conpo elternante of igucl à frequência ciclotrônica do fon de massa m, as soluções (l-ll) e $(1-12)$ se convertem $\mathrm{em}$

$$
\begin{aligned}
& v_{x}(t)=\frac{q E_{0} t}{2 m} \operatorname{sen} w_{c} t+v_{L}^{0} \operatorname{sen}\left(w_{c} t+\gamma\right) \\
& v_{y}(t)=\frac{q E_{0} t}{2 m} \cos w_{c} t+v_{L}^{0} \cos \left(\omega_{c} t+\gamma\right)
\end{aligned}
$$

A trajetbria do lon neste problema, ou mais pròriamente - raio da trajetória curvillnea, pode ser obtido simplesmente a partir das expressões para $v_{x}$ e $v_{y}$ na equação (1-3).

$$
\begin{aligned}
& \text { Qucindo } \quad \omega_{1} \neq w_{c} \\
& v_{1}=\sqrt{v_{x}^{2}+v_{y}^{2}}=\frac{q E_{0}}{m\left(v_{x}-w_{1}\right)} \operatorname{sen} \frac{\left(w_{x}-w_{x}\right)}{2} t+v_{b}^{0} \\
& r=\frac{c E_{0}}{H}\left|\frac{\operatorname{sen} \frac{\left(w_{1}-w_{2}\right) t}{2}}{w_{c}-w_{1}}\right|+\frac{m c v_{1}^{0}}{q H}
\end{aligned}
$$

Quando $w_{1}=w_{c}$,

$$
r=\frac{c E_{0} t}{2 H}+\frac{m c v_{2}^{0}}{q H}
$$

c o raio da trajetoria circular cresce lincamente com o tempo, num movimento espiral.

Na condição de ressonência, $\left(\omega_{1}=\omega_{t}\right)$, e de acôrdo com as equações (I-I4) e (I-I6), o lon é acelorado pelo compo elétrico altomante, e consequentemente $a$ energia cinetica do Ion aumontará com o tompo. Êste acréscimo de energia cinética pode ser estudado ern têrnos de potência absorvida pelo fon do campo elétrico altomante.

$$
\text { Potência } A\left(\omega_{1}\right)=\left\langle\frac{d}{d t}\left(\frac{1}{2} m *^{2}\right)\right\rangle=m\left\langle\vec{v} \cdot \frac{d \vec{v}}{d t}\right\rangle
$$




$$
A\left(w_{1}\right)=m\left\langle\vec{v} \cdot\left(\frac{q \vec{E}_{1}(t)}{m a}+\frac{q(\vec{v} \times \vec{H})}{m c}\right)\right\rangle=q\left\langle\vec{v} \cdot \vec{E}_{1}(t)\right\rangle
$$

Os colchetes nas equaçócs $(1-17)$ e (1-18) indicarn 0 velor médio de $\vec{\nabla} \cdot \vec{E}_{1}(t)$ com rolagẽa no tempo.

Desenvolvendo o produto escalar en têmos da velocidade do 1on, obtêm-se

$$
\begin{array}{r}
A\left(\omega_{2}\right)=\frac{q^{2} E_{0}^{2}}{4 m}\left\langle\cos \omega_{1} t \operatorname{sen} \omega_{1} t-\operatorname{sen} \omega_{1} t \cos \omega_{t} t\right\rangle \frac{1}{\left(u_{t}-\omega_{1}\right)} \\
A\left(w_{1}\right)=\frac{q^{2} E_{0}^{2}}{4 m\left(u_{c}-\omega_{1}\right)}\left\langle\operatorname{sen}\left(\omega_{c}+\omega_{1}\right) t\right\rangle
\end{array}
$$

Para colcular o velor médio com relaģão a tempo considera-se o comportamento da equecão (1-20) nun intervalo de tempo definido. Êsse intervalo de tempo pode ser escolnido entre $t=0$ e $t=\tau$, onde $\tau$ represente o tempo que 0 fon fica sob a influencia do canpo elétrico. Del então, calcula-se a potência absorvida em função da frequência do compo altemente.

$$
\begin{gathered}
A\left(\omega_{1}\right)=\frac{q^{2} E_{0}^{2}}{4 m\left(\omega_{\varepsilon}-\omega_{1}\right)} \frac{1}{\tau} \int_{0}^{\tau} \operatorname{sen}\left(\omega_{c}-\omega_{1}\right) t d t \\
A\left(\omega_{t}\right)=\frac{q^{2} E_{0}^{2}}{4 m\left(\omega_{c}-\omega_{y}\right) \tau}\left[\frac{1-\cos \left(\omega_{t}-\omega_{1}\right) t}{\left(\omega_{t}-\omega_{1}\right)}\right]
\end{gathered}
$$

A potência absorvide of de interesse om certas aplicacones da ressonencia ciclotrônice de lons, e sua utilidade será ilustrada posteriormente. 


\section{I.3 Aplicacão qufmice dos princlpios do ciclotron}

Os princlpios Ifsico-mateméticos estebelecidos nas primeires pefinas deste tese constitucm os principios bésicos do funcionamento de un ciclotron, cujo uso es ben conkecido em flsica nuclear. Porkm, ôstes princlpios que sño utilizados para acelorar projeteis nucleares a altos velocidades, contên uma serie de possfveis aplicacõos para estudos quinicos.

Primeirmente, êstes princlpios poderiom ser usados pare construir ure espectrômetro de massas, sc a substêncie a ser estudada fôr previemente ionizada, e molisada on seguida ein função da frequencia ciclotrônica dos fons presentes. Tal corno foi estabelecido na equação (1-4), egta frequência depende inversamente da grandeza $\mathrm{m} / \mathrm{q}$.

Jin segundo lugar, êstes princlpios poden ser usedos cono um ciclotron qufnico para acelerar lons a onergies definida, e estudar reações qufricas produzides por êstos lons em função de sue onorgze cinétice translacional. 


\section{CAPÍPULO II}

ESPICTROSCOPIA DE RESSONANCIA CICIORRONICA DE IONS

\section{II.I Resumo histórico}

A princire aplicação química dos princlpios expostos no primeiro capftulo foi feita por Fipple, sommer o thomas ${ }^{3}$ na construção de un espoctrônetro de massas chenado onegatron. 0 método experimental consistia fundamentolmente em produzir lons monovelentes por bombardeio cletrônico num cempo mgnetico, c aceleré-los seletivanente de acôrdo com as sues massas por reio de um oscilador de radiofroquencie varível, operando na frequência ciclotrônica do lon selecionado. Os fons acelerados em orbite espiral, do tipo ilustrado pela ecuação (1-16) erom detectidos num coletor de lons colocedo a uma certe distência da regìĩo de formação dos 1ons. Êste método demonstrou ser muito vantajoso no que se refere à alta sensibilidade do ape-rêlho para detectar lons on bnixe concentraça na onostra gasosa. Porén, o poder de resolução do onegetron é forelmente muito inforior a outros tipos de espectrômetros de massa. Consequentemente, seu uso como espectrômetro de messas tem sido relativamente limitado. Alguns dos detelhes preticos do omogetron podem ser encontredos no Iivro de Kiser. 4

Una versão mais aprinorda dêste aparôlho foi consitruide por lobschall, Grahar a Malono ${ }^{5}$, oproveitando a potêncio absorvida do oscilador de radiofrecuência para detecter os lons de massa $m$ on ressonencia. Un dos aspectos guinicos mais interessantos desta versão \& o benpo reletivemente longo de permenência do fon na cela do espectrôtritro, possibilitendo que os lons sofrom una serrie de intorações físicas e quínicas 
com outras moleculas presentes na cela. Os avtores uscrm o aparêtho de ressonência ciclotrônice pere o estudo des seções de choque para espalhamento entre lons e moléculas, a portir da largura das linhas de absorção.

Finalmente, en 1966 Baldeschwieler e seus colaboradoros (referencias 6 a 8 ) desenvolveram a técnica hojo chamada de espectroscopia de ressonância ciclotrônica de lons, cue serviu para introduzir um metodo revolucionário no estudo das reaços entre lons e noleculas. As modificaçoes introduzidas no metodo experimental fizeram com que o método ficasse muito somlhonto d̀ têcnicas de ressonância magnêtica.

As proximas partes dêsto capitulo visam explicar a parte instrumental da RCI, assim como outros aspectos físicos de importância, não mencionados no prinoiro capitulo mas necessórios para a compreensão dos resultodos experimentuis.

\section{II.2 Espectrômetro}

o espectrômetro de ressonêncio ciclotrônica de lons usado nesto tese f fundanentelmento o aparêlho produzido pela Vericn Associates (Palo Alto, U.S.A.), sob o nome de Syrotron. O espectrômetro é suficientenente versátil, podendo ser modificado de acôrdo com as experiências a serem roclizada.s.

A figura 2-1 apresenta um oscueme geral do espectrô.metro usado, enquanto que a figura 2-2 descreve a cola cm detalhes. A cela do espectrômotro usada nas experiências das aninas tem $2,54 \mathrm{~cm}$. de altura, $2,54 \mathrm{~cm}$. de largura e $12,7 \mathrm{~cm}$. 
do comprimonto. Dla se ache localizada ontre os polos de um oletrimã capaz de produzir compos magnoticos ontro 0 o 14.000 gauss. A cele csté contide num tubo retangular con una ontrada para a anostrc gasosa, e ligade a uno bombe iônica que mantóm a cela a una pressão de $10^{-8}$ torr. quando o eparêlho não estê sendo utilizado.

A cela ilustrada na figurc 2-2 possue três regiões definias. Na prineira parte da cela (regiẽo fonte), o gés \& subnetido a um bombardeio de elétrons onitidos por um Iilnmento. A cnergia dos eletrons, assim como a intensidade da corrente eletrônica são controlados de coôrdo com as exiş̂ncics experimentais. A corrente de emissão é gerelmente regulado. no máxino a 0,8 microamperes para manter un grau de ionização relativanento baixo.

Os lons formados ne prineira rogião da cela estẽo sujeitos 20 canpo magnético e as campos eletricos constentes aplicados nes porodes. A voltagem chanada do aprisionamento no esquema, \& variável de 0 a 2 volts, e tem por objetivo prineipal repelir os lons positivos (ou negctivos se fôr o caso) da região proxima às paredos latorais da cola. isse. voltagen faz com que os lons positivos estejom concontrados no meio da cela, enquanto que os lons de sinol oposto sojam recolhidos nas paredes.

A voltagen aplicade na parode superior de prineira região, e identificada como voltazon de arraste da rogiño fonte nos efreficos, \& variével ontre 0 e 1 volt. Seu objetivo rpincipal e arrastar os lons para a segunda regiõo por meio de um novimento que pode ser facilmente explicedo pelas equaçoos derivadas no prineiro capltulo, se o campo 


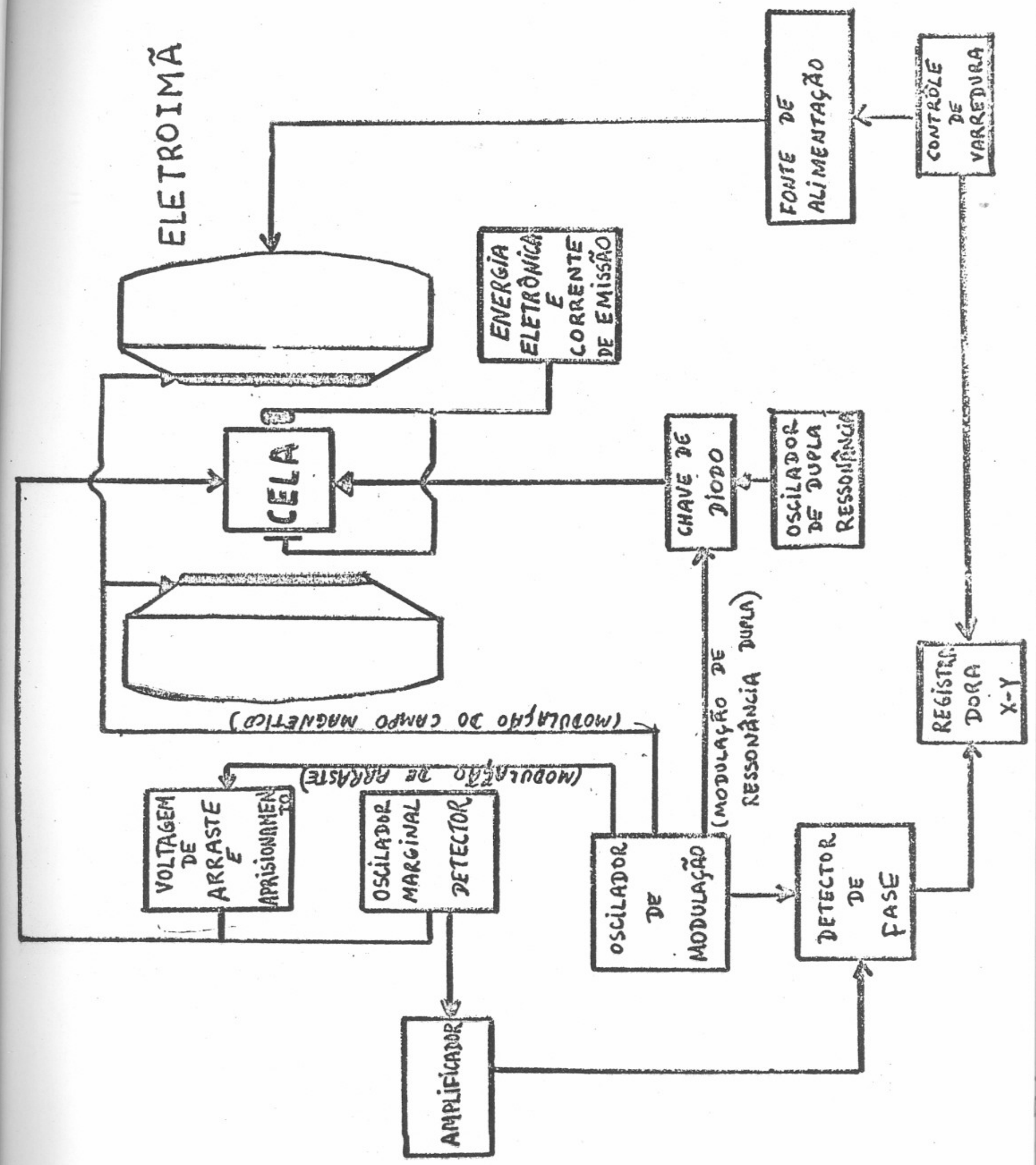

FIGURA 2-1

Tspectrômetro de ressónência ciclotrônica de lons. 


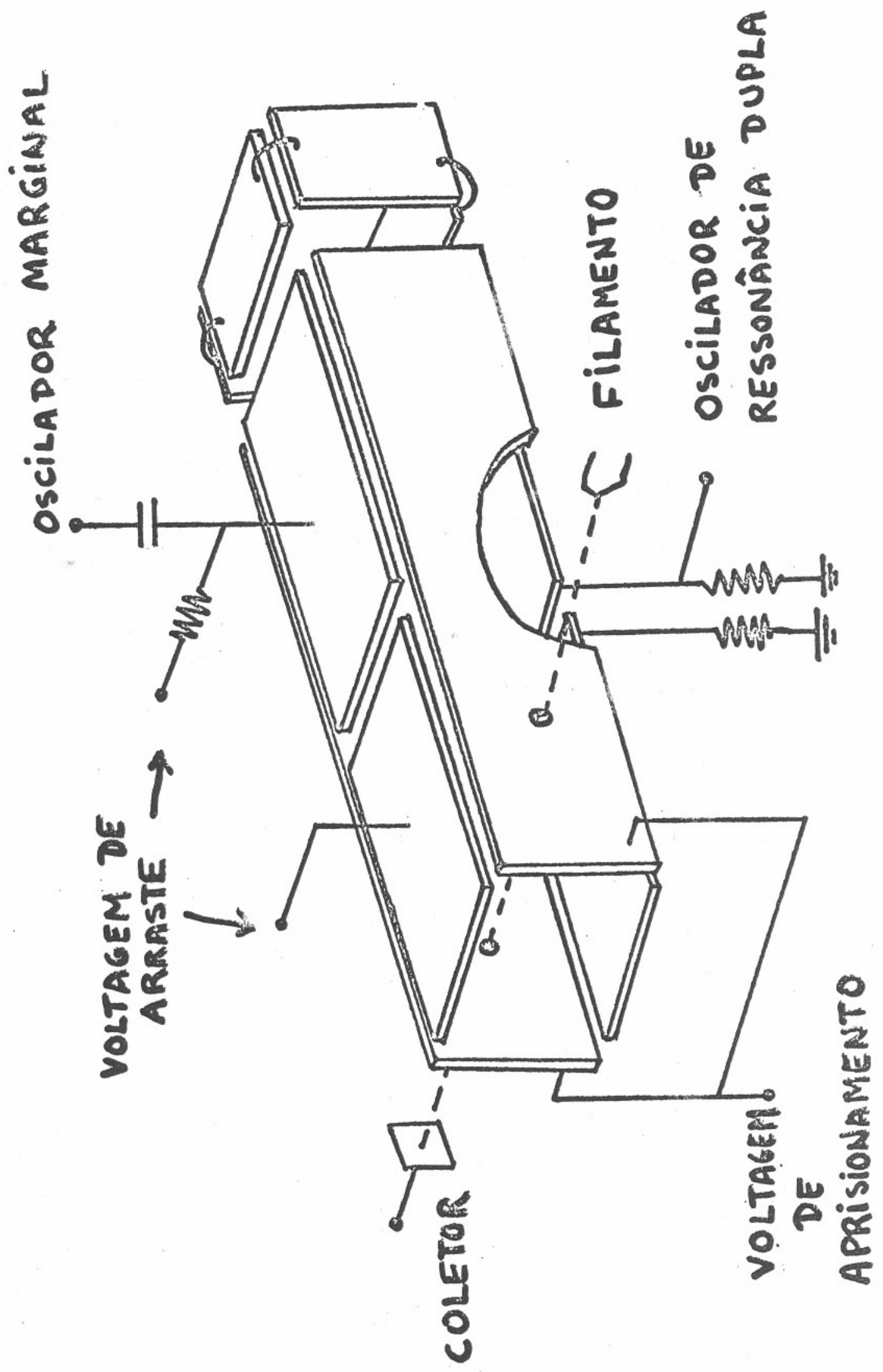

FIGURA 2-2

Cela do espectrômetro de RCI em detelhes 
el6trico altomante \& substituido por um compo clétrico constante. Os datalhos mateméticos sño explicados no itom II.3.

Na segunda região da cola (região analisadora), alóm das voltagens constontes aplicedas nos paredes de cela, os lons estão sujeitos a.os efcitos de um oscilador de rodiofrequência. o oscilador de radiofrequência usodo no espectrômetro é do tipo usado em ressonância magnética nuclear, muitas vêzes denominado como "oscilndor marsinel", 9-11

A tercoira perte da cola recolho os fons e mede a corronte iônica.

A cela faz parte do circuito do oscilador como um condensador. Conseqüentemente, uma absorção de onoræia por parte dos fons dentro da cela se reflete no nivel de oscilaç̃o do oscilador. Este mudança no nível de oscilação é detecteda e amplificada.

A amplificação dêste fenômeno é muito maior se $a$ absorção de enorgia é modulada $c$ a deteç̧ão b feita num amplificador de fase (lock--in amplifier ou phese sensitive detector). Esta técnice é ceraterística de tôdas as formas espectroscopia de radiofrequência. ${ }^{12}$

Os espectros de ressonância ciclotrônica deste tese foram obtidos modulando o campo magnético com uma frequência de $38 \mathrm{~Hz}$, usando-se esta frequência como referência no amplificador de fase (Princeton Applied Rescurch, modêlo JB-5). No esquema do espectrômetro, existe a possibilidade de moduler a voltagem do arraste, mas este altemativa não foi usado. Algumas experiências recentes têm demonstrado a possibilidade ae modulação de energia oletrônica ${ }^{13}$, e ainda modnlnoño de corrente de emissão do filamento. ${ }^{14}$ 
Os espectros form rogistrados montondo a froquencia do oscilador "maxginal" fixada on $153,6 \mathrm{kHz}$, e, varrendo 0 campo megnético. A frequência escolhida como fixa es tal que a massa do Ion monovalente observado num espectro corresponce a $\mathrm{H} / 100$, onde $\mathrm{H}$ e o campo magnético em gauss.

Un espectro t1pico de ressonâncic ciclotrônica é apresentado na figura 2-3. Este espectro obtido com modulaşa do campo magnetico, dá origem à obsemaçõo na rogistradora da primeira derivada da Iinha de absorção. Os motivos que levam ao registro da primeira derivada, assin como as caraterlsticas desta linha em função da modulação são discutidos no livro de Poole. 15

Um segundo oscilador convencional de radiofrequência. e Iigado na parede inferior da seguna região da cela. Aste oscilador é utilizado nas experiências de ressonência dupla, e para êsse fim foi empregado um oscilador Hewlett-Packard, modêlo 6515, de frequência varífvel. Âste segundo oscilador pode ser acionado de forma continua, ou pulsado periodicamente na voltagem de saía. ísta pulsação constitui mais uma tócnica de moaulação.

\section{3 Consideracões préticas sobre o movimento dos fons na celo do espectrometro}

As equações derivedas no prineiro capitulo considerarä a situação ideal de un fon isolado num campo magnético, $e$ submetido a um campo de radiofrequência. Porém, os detalhes préticos da constmęão da cela, apresentas situcções mois complicadas do quo as até aqul consideradas. 


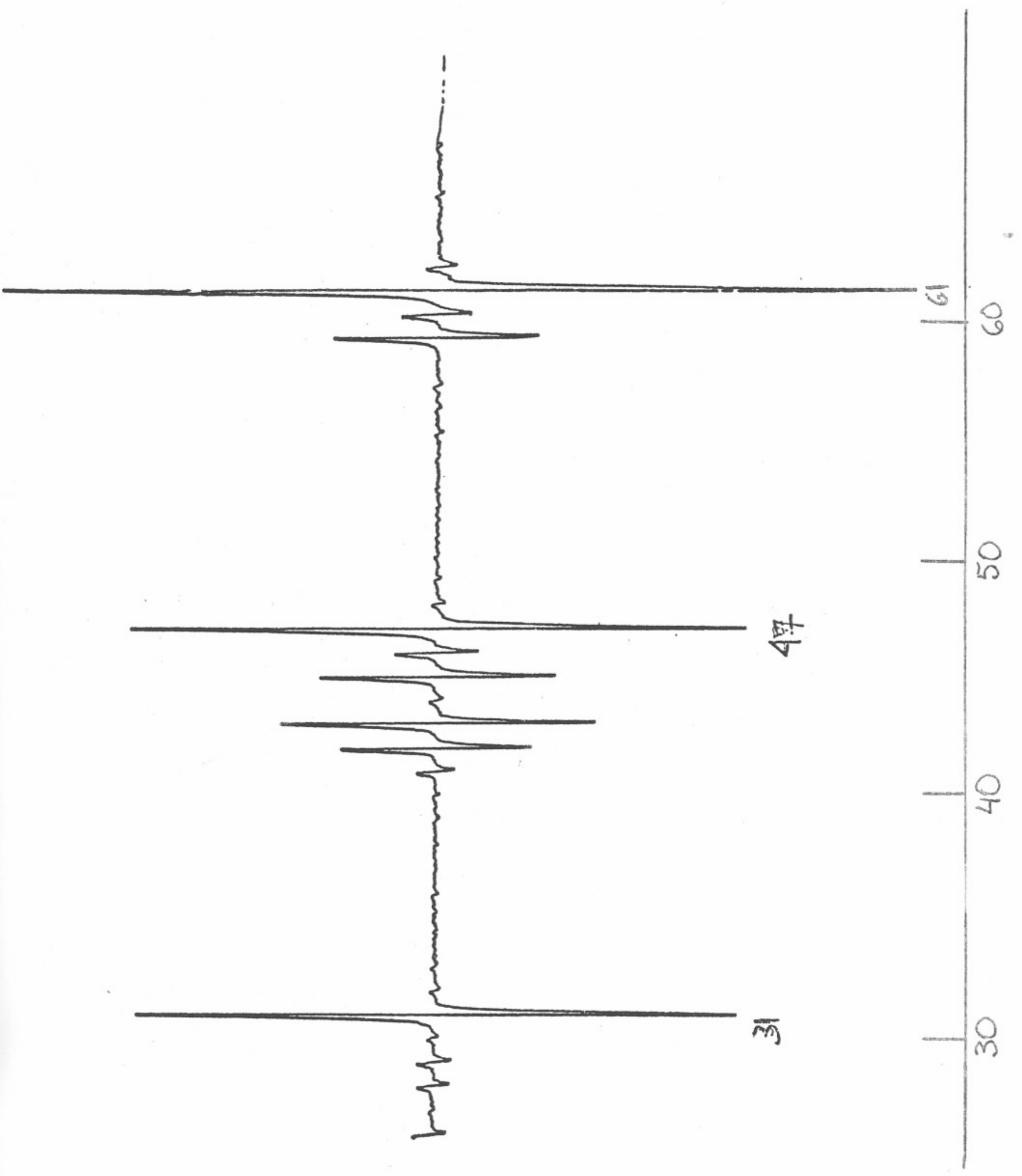

FIGURA 2-3

Espectro de RCI de uma mịtura de etanol e propnol obtida a $16 \mathrm{eV}$ a uma pressão de $10^{-5}$ torr. Ispectro obtido modulnndo o compo megnetico. 
(a) VOLIAGII DE APRISIONANTHTO

imbora seja facil compreender quolitativonente a função do potencial aplicado nas paredes laterais da cela, êsse potencial produz alterações no movimento do 10n. A presença dêste campo elétrico tem sido analisada por Andors ${ }^{16}$ e Becuchamp. ${ }^{17}$ A conclusão mais importente destes estudos foi a comprovação de uma oscilação lateral dos lons repelidos devido a um poço de potencial no meio da cela. A ajlicação desta oscilação lateral para auxiliar certos estudos tem constituido una extensão muito elegante da RCI.18,19

Esta oscilação lateral do fon afeta a frequência ciclotrônica efetiva do movimento. Consequentemente, o campo magnético necessário para observar a ressonencio de um certo lon depende da voltagem de aprisionamento.

De acôrdo com os célculos de Beauchamp ${ }^{17}$

$$
u_{\text {eff }}=\left(w_{c}^{2}-4 q V_{T} / m d^{2}\right)^{1 / 2}
$$

onde

$\omega_{e}$-- frequência ciclotrônica do lon

$\mathrm{V}_{\mathrm{T}}$-- voltagem de aprisionamento

d -- distancia entre as paredes laterais.

A condição $\omega_{c}^{2} \gg 4 \mathrm{qV}_{\mathrm{T}} / \mathrm{md}^{2}$ é sempre satiisfeita nas experências.

(b) VOLTAGJM DE ARRASEE

A função da voltagem de arraste pode ser compreendida matematicanente resolvendo a equação diferencial apropriada. Para estudar isoladamente os efeitos desta voltagem, os outros campos elétricos (oprisionamento o radiofroquência) são ignorados. 
Nessa condições, o movimento do fon dentro da cela fico. representado pela equação (2-2)

$$
m \frac{d \vec{v}}{d t}=\frac{q}{c}(\vec{v} \times \vec{H})+q \vec{E}_{A}
$$

Deve-se lembrar que $\mathbb{E}_{A}$ na região fonte dos lons, e $\mathbb{E}_{A}$ na região de detecção são controlados independentemente. Dal, cada região estaria caraterizada por uma equação (2-2) diferente, dependendo do valor de $\mathrm{E}_{\mathrm{A}}$.

Se o sistema de coordenadas definido na figura I-I fôr adotado, o campo elétrico $E_{A}$ terá sua componente na direção do vetor unitário $\vec{j}$. Desenvolvendo a equação (2-2) em suas componentes, obtém-se

$$
\begin{aligned}
& \frac{d v_{x}}{d t}=v_{y} w_{c} \\
& \frac{d v_{y}}{d t}=-v_{x} w_{c}+\frac{q}{m} E_{A}
\end{aligned}
$$

Diferenciando a primeira equação com relação no tempo, e substituindo $d v_{y} / d t$ por seu valor na segunda equação, tem-se

$$
\frac{d^{2} v_{x}}{d t^{2}}=-\omega_{c}^{2} v_{x}+\frac{q w_{c}}{m} E_{A}
$$

A solução da equação (2-4) é simplesmente

$$
v_{x}=v_{d}^{0} \cos \left(\omega_{c} t+\gamma\right)+\frac{g E_{A}}{\omega_{c} m},
$$

ou substituindo $\omega_{c}$

$$
\begin{aligned}
& v_{x}=v_{+}^{0} \cos \left(u_{c} t+\gamma\right)+\frac{c E_{a}}{H} \\
& v_{y}=v_{1}^{0} \operatorname{sen}\left(\omega_{c} t+\gamma\right)
\end{aligned}
$$


com $y$ representando a defasagem da velocidado com relação ao sistema de coordonadas adotado no $t=0$.

Nesta. orientação de campo magnetico e eletrico observa-se que a velocidade do fon tem uma componente neta na direção $\underline{x}$, dada pelo segundo tômo da expressc̃o de $v_{x}$. îsse têrmo pode ser identificado como a velocidade de arraste, e seu valor tip̣ico será da orden de $10^{3} \mathrm{~cm} / \mathrm{seg}$.

A representação gráfica do movimento periódico derivado destas equações e ilustrada na figura 2-4. (Ver tombém Refis. 19 e 20).

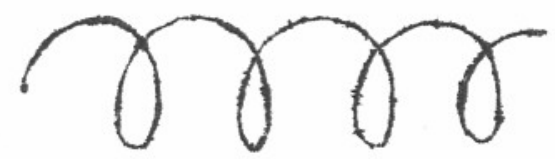

Figura 2-4

Uma consequência inportante dos campos elétricos analisados nas partes (a) e (b) dêste 1ttm, é que quando o efeito combinado dos dois e considerado, chega-se a um compo potencial complicado. A presença dêsse potencial complicado cria incertezas na avaliação de certas grandezes cinéticas, e constitui indiscutivelmente uma limitação no método da RCI na aplicação quantitativa a problemas cinéticos.

(c) WFITO DOS CHOQUES DOS IONS COM MOLEUCUAS NUUTRAS

A absorção de energia observada experimentalmente é devido a un conjunto de lons com uma certa distribuição estat1stica de suas propriedades da tronsporte. Essa distribuiç̃o diviuistica será ofetada pelos choques dos lons com outras partículas. 
Un tratamento estatistico dêste efeito foi considerado pora um conjunto de elétrons por Kelly, Margeneu e Brow ${ }^{21}$ usando a equação de transporte de Boltmann. Paro o coso da interąão de fons com moléculas noutros, Beauchamp desenvolveu. a equação de Boltzmann, e os resultados tobricos foram verificados experimentalmente. 22

Como aquí so interessa ilustrar o efeito çeral sôbre un conjunto do fons ne cela, o modêlo escolhido pode ser o mais simples possível. Assim, an luger de considerar a equeção de Doltmann, vanos considerar o simples caso de un número $\underline{n}^{+}$de lons de massa mn e volocidado média $\langle v\rangle$, interagindo com un número $\mathbb{N}$ de moléculas neutras, (N $\gg n^{+}$). Esta interação vai se manifestar na cela como choques ontre estas duas espécies, já que o lon pemanece na cela um tenpo relativanente longo om rolação à frecuência média de colisões. O tipo de interação considerada vai ser restringido a troca de energia translacional entre íns e moléculas neutras, desprezando então as possibiIidades de choques inelésticos, reacões quínicas ou troca de corge ontre fon e molécula neutra.

Com as restricções inpostas no parágrafo antorior, devonos nos preocupar com a situação na qual êstes choques sc̃o de importância. Histes choques interrompen o movimento peribaico do fon na cola do espectrômetro, e portanto afetam a.s equações consideradas nas partes (a) e (b) dêste ítem assim coino a equação considerada no ítom I.3 . Êste últino caso vai ser considerado isoladmento, ignorando (a) e (b), porcue o que interossa é a influência dos choques elésticos no processo de absorção de cnergic de rediofreguencia. 
A equação de movimento de um lon "mćdio" (íon com propriedcdes de transporte correspondentes c̀ media cstatistica delas) na presença de um campo magnótico, e un canpo elótrico de radiofrequência, será semelhante ¿ equação (1-5), com a adição de um tôrmo responsável pelos choques. Se consideramos a situação na qual o lon esté om ressonência ou quase en ressonância $\left(\omega_{1} \nsucc \omega_{c}\right)$, as colisões servirão para rolaxar - excesso de energia translacional do 1on, absorvida da fonte de radiofrequência. Sob essas condiçõos, a equação (2-7) deve ser considerada como uma equação fenomonológica.

$$
m \frac{d\langle\vec{v}\rangle}{d t}=\frac{q}{c}(\langle\vec{v}\rangle \times \vec{H})+q \vec{E}(t)-\nu_{c} m\langle\vec{v}\rangle
$$

0 último têrmo aparece como um tôrmo de amortecimonto na. equação de movimento, e so refero à transferência de momento linear para as moléculas neutras com uma eficiência V , definida sem muito rigor como frequência media do colisão eléstica entre fon e molecula.

Esta equação pode ser desdobrada de maneira similar àquela empregada para resolver as equações (1-8).

$$
\begin{aligned}
& \frac{d\langle v\rangle_{x}}{d t}=\frac{q E_{b}}{2 m} \operatorname{sen} \omega_{i} t+\omega_{c}\langle v\rangle_{y}-v_{c}\langle v\rangle_{x} \\
& \frac{d\langle v\rangle_{y}}{d t}=\frac{q E_{0}}{2 m} \cos \omega_{y} t-\omega_{c}\langle v\rangle_{y}-\psi\langle v\rangle_{y}
\end{aligned}
$$

A solução destas equações diferenciais acopladas \&

$$
\begin{aligned}
& \langle v\rangle_{x}=\frac{q E_{0}}{2 m} \frac{1}{v_{c}^{2}+\left(\omega_{0}-w_{2}\right)^{2}}\left[\left(\omega_{c}-\omega_{3}\right) \cos \omega_{1} t+v_{c} \operatorname{sen} \omega_{1} t\right] \\
& \langle v\rangle_{y}=\frac{q E_{0}}{2 m} \frac{1}{v^{2}+\left(w_{t}-\omega_{1}\right)^{2}}\left[r \cos \omega_{j} t-\left(\omega_{k}-\omega_{1}\right) \operatorname{sen} \omega_{1} t\right]
\end{aligned}
$$

Bstas expressões ignoram as condiçõos iniciais devida a situação particular na qual a equação (2-ô) é oplicóvol. 
Outrossim, as soluções (2-9) se referem a um tempo $t \gg \frac{1}{t}$, de maneira que as múltiplas colisões fazem com que os lons não sejam afetados pelas condições iniciais.

Conhocendo estas soluções, calcula-se a potência absorvida por um conjunto de $\underline{n}^{+}$Ions através das equações $(1-17)$ e $(1-18)$

$$
A\left(\omega_{1}\right)=q n^{+}\left\langle\langle v\rangle_{x} \frac{E_{0}}{2} \operatorname{sen} \omega_{1} t+\langle v\rangle_{y} \frac{E_{0}}{2} \cos \omega_{1} t\right\rangle
$$

Como a potência absarvida interessa so quando $\boldsymbol{w}_{1} \simeq \boldsymbol{w}_{\boldsymbol{q}}$, - tôrmo multiplicado por $k$ será a componente de importânoia na expressão de velocidade, enquanto que o outro tamo pode ser desprezado. Nessa condições

$$
A\left(w_{2}\right)=\frac{n^{+} q^{2} E_{0}^{2}}{4 m} \frac{v_{4}}{v^{2}+\left(w_{k}-w_{1}\right)^{2}}
$$

A conclusão dêste tratamento ê cue sob condiçõos nas quais a equação (2-7) é válida, a linha de absorção do espectro de ressonn̂cia ciclotrônica terá uma forma Iorentziana, caraterlstica de outros tipos de espectroscopic (ressonência magnétjca e elétrica). Observa-se cue a partir da largura da linha de absorção soria possivel calcular a frquência méaia de colisão elástica, se êste fôsse o único processo de interação entre fona maléculas.

Ura tratamento mais rigoroso e mais geral, incorporando outros possiveis processos entre lons e moleculas, e detalhado no trabalho de Beauchomp. 22 
II.4 Efeito de ressonência dupla e acoplamento dos movimentos entre lons

O espectro de massas obtido com o aparêlho de rossonência ciclotrônica apresenta em geral una série de picos de absorção, a medida que o campo magnetico \&́ varrido. Ŝsses picos correspondem a lons de massa diferente como se observa na figura $2-3$.

Os Lons detectados no espectrômetro sẽo produzidos por diferentes processos: 1) ionização direta da mostra gasosa. pelos clétrons, 2) frogmentação dos lons prinérios produzidos coin excesso de energia interma, o 3) reação de un ion primário com uma molécula neutra para produzir un fon secundśrio, e processos similares devido a fons secundérios.

Considere-se o caso de uma reação entre íon $\mathrm{I}^{+} \mathrm{e}$ ume. molocula neutra A pare produzir um outro fon $\mathrm{J}^{+}$.

$$
\mathrm{I}^{+}+\mathrm{A} \rightarrow \mathrm{J}^{+}+\mathrm{B}
$$

0 movimento dos fons $\mathrm{I}^{+}$e $\mathrm{J}^{+}$na cela do espectrônetro jode ser representado pelas equações (2-11), se as voltogens constantes das paredes são desprezadas

$$
\begin{aligned}
& \left.\frac{d\left\langle\vec{u}_{i}\right\rangle}{d t}=\frac{q E_{1}}{m_{i}} \operatorname{sen} \omega_{1} t \vec{\imath}+\left(k \vec{v}_{i}\right\rangle \times \overrightarrow{u_{i}}\right)+ \text { têrmos de smortecimento } \\
& \frac{d\left\langle\overrightarrow{w_{j}}\right\rangle}{d t}=\frac{q E_{1}}{m_{j}} \operatorname{sen} \omega_{j} t \vec{i}+\left\langle\left\langle\overrightarrow{w_{j}}\right\rangle \times \vec{w}_{j}\right\rangle+\text { têrmos do amortacinento }
\end{aligned}
$$

Se o campo magnético fôr ajustado de tel mancira que - Ion $\mathrm{J}^{+}$esteja cm ressonância ( $u_{j}$ ), o efeito do conpo clótrico será desprezivel para o fon $\mathrm{I}^{+}$de acôrdo com as equaçõos (I-II) e (I-12). Assim, a trajetoria do ín $I^{+}$ será pràticanonte circular, enquanto que a trajetória do ín 
$\mathrm{J}^{+}$o a potência por $\widehat{l} \mathrm{Ie}$ absorvide estarão represcntados pelas equações derivadas na parte anterior. Como $\mathrm{I}^{+}$c $\mathrm{J}^{+}$estẽo ligados por uma reação qufrnica, as suas equações do movimento estarão acopladas atravós do tômo de amortecimonto que leva. om consideração a interrupção do novimento de $\mathrm{I}^{+}$con $\varepsilon$ conscquente formação de $\mathrm{J}^{+}$. Isto implica om que uma mudença em $\left\langle v_{i}\right\rangle$ efetará $\left\langle v_{j}\right\rangle$, se a constante de velocidode da reação depender da velocidade do lon reagente.

Experimentelmente, a velocidade do fon $\mathrm{I}^{+}$pode ser mudada com ajuda de un segundo osciledor de rodiofrequência sintonizado na frequência ciclotrônica do fon $\mathrm{I}^{+}$. Assim, - espectro de ressonância ciclotrónic do fon $\mathrm{J}^{+}$obtido com un compo magnético H e frequôncia do oscilador marginal $\boldsymbol{u}_{1}$, \& perturbado pela presença do segundo oscilador de frequêncie

$$
\omega_{2}=w_{i}=\frac{q H_{6}}{m_{i} 6}
$$

que \& ligado na parede inferior da cela. (Vor figura 2-2).

Esta tóchlec de ressonẩncte dupla s muito especifica para indicar a relação entre lons observados no espoctro. Isto pemite então identificar quais são os fons precursores de um fon en particular, um aspecto que precisa ser postulado cm espectros de massa comung. Âste método permite esencialmento construix a árvoro genealógica de un lon. 


\section{ESTUDO DA BASICIDADE REIAIIVA DAS AMTHAS}

\section{III.I Introducão}

Os trabalhos recontes de Brauman e 31 air 23,24 sôbre a acidez relativa de compostos orgânicos a partir dos dados obtidos por ressonância ciclotrônica de fons, estinularan - estudo da basicidade relativa das aminas na fase gasosa. Este tipo de problema \& fundarontel por ter relação direta com os conceitos mais tradicionals da quimica, e por tratar de ume. das propriedades mais fascinantes dos compostos químicos. 25,26

Logicamente, a doteminação das propricdades relatadas nesta tese tom sido objeto de múltiplas pesquisas atravs dos anos. Os resultados dessas pesquisas podem ser encontrados cm tabelas convenientes 27 , rosumidas nos valores da constinte de dissociação cm soluções aquosas. Poróm, qualquer estudo na fase Ilquida \& Iimitado pelo simples fato de que a propriedado de acidez ou de basicidade de um composto esté intimamente ligada 20 meio no qual essa propriedade é deteminada. Assim, os resultados tabelados na. Ref. 27 dependem implicitmente da solvateção do composto e dos lons dissociados na fase Ilquida. Êstes ofeitos de solvatação são indiscutivelnente de muita importência, porque na realidade muitas vêzes interessa a propriedade de basicidade em detominado moio no qual uma reoção ou um fenômeno estão sendo absorvados. Entrementes, êstes efeitos de solvatoção criam dúvidas sôbre as propriedades intrlnsocas de basicidode, e a relação entre estrutura nolecular e comportanento quimico. 
A fanília das aminas é particulamente intorossonto já que existe uma grande voriedade delas entre as mais simplos, incluindo os primérias, secundárias e torciérias. Sou estudo permite avaliar os efeitos de grupos alquile localisados porto do centro responsável pola basicidade das aminas.

Alguns estudos prelininoros desto netureza, cbrongindo as aminas o outros compostos na fase gasosc, form realizados por Munson 28 fazendo uso do espectrometria do massas. Bmbora, os resultados de Iunson sojan confimndos pelos ostudos opresontados nôte trabalho, assim como pelas medidas do Braumen - Blair24, a intorpretação dosses resultados prolininaros esté bascada om argumentos limitados.

\section{III.2 Caratoristica geral dos espoctros de RCI das aminas}

O espectro de ressonância ciclotrônica simplas das aninas aliféticas é semelhonte aos espectros de massas caraterizados por Gohlke o lic Iafferty. 29 As intensidades rolativas dos picos diferem daquelas do artigo mencionodo, devido às cornteristicas do espectrômetro, e sobrotudo pola presença do picos atribuídos a produtos de reções entre 1ons primérios c molsculas noutras.

A figura 3-1 ilustra o espectro de ressonência ciclotrônice de $\left(\mathrm{CH}_{3}\right)_{3} \mathrm{C}-\mathrm{NH}_{2}$ registrado a uma pressão de $10^{-5}$ torr. o a uma cnorgic elotrônica nominal do 76 ov. Os picos mais prominentes nesto espectro corrasponden a
$\mathrm{m} / \mathrm{q}$
41
$\mathrm{C}_{3} \mathrm{H}_{5}+$
$\mathrm{m} / \mathrm{q} \quad 58$
$\left(\mathrm{CH}_{3}\right)_{2} \mathrm{CHH}_{2}{ }^{+}$
$\mathrm{m} / \mathrm{c} \quad 74$
$\left(\mathrm{CH}_{3}\right)_{3} \mathrm{CNH}_{3}{ }^{+}$ 


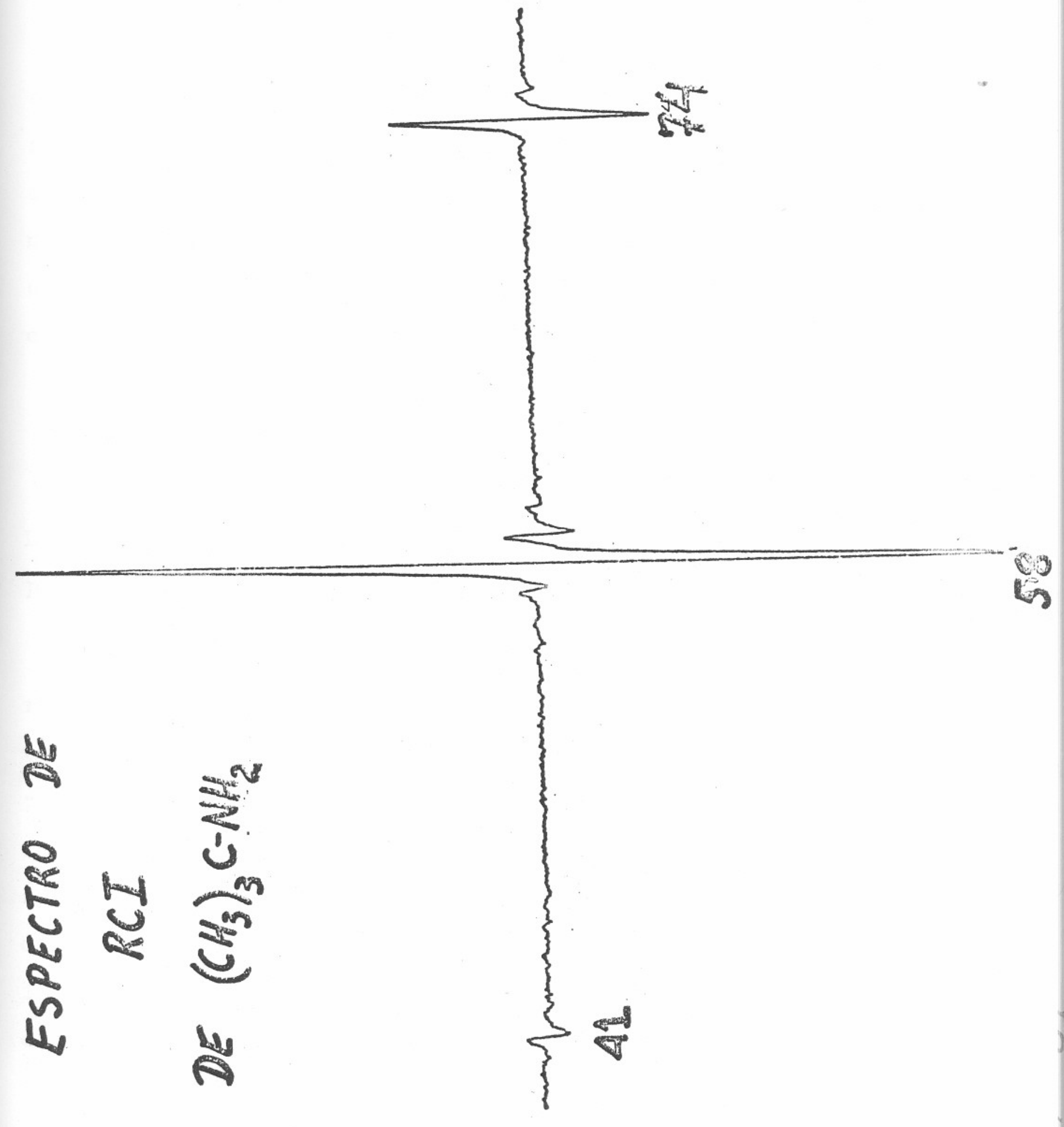

PIGURA $3-1$ 
Os dois primeiros lono correspondem nos picos observados com maior intonsidade no espoctro de massos de $\left(\mathrm{CH}_{3}\right)_{3} \mathrm{CHH}_{2}$. o torceiro Ion, de messe 74, deve ser o produto formado por uma reação na cela do espectrômetro ontre um lon primério e uma nolscula neutra.

Os espectros de outras aminas apresontan corotorlsticas semelhontes dis observadas neste caso, com picos dovido a fons secundérios. Os picos correspondentes das egpécies protonadas, cono aquela ropresentada pelo lon de massa 74, são particularmente interessantes, ja que a formeção dessas espécies depende da propriedade tomodinâmica chanada ofinidado protônica ${ }^{30}$, definida pela equação $(3-1)$.

$$
\begin{gathered}
\mathrm{H}+\mathrm{H}^{+} \longrightarrow \ldots \mathrm{HH}^{+} \\
\text {A.P. (N) }=\Delta \mathrm{H}_{\mathrm{f}}(\mathrm{H})+\Delta \mathrm{H}_{\mathrm{f}}\left(\mathrm{H}^{+}\right)+\Delta \mathrm{H}_{\mathrm{f}}\left(\mathrm{IH}^{+}\right)
\end{gathered}
$$

Esta afinidade protônica, associada com o efoito de entropia para a reação (3-1), contribui para a basicidade da molécula M.

istes lons protonados apresenton um aspecto muito interessante no espectro de RCI de uma nistura aproximadamente equinolar de duas aminas. Isto \& ilustrado na figura 3-2. para. o espectro de ume mistura de $\mathrm{CH}_{3} \mathrm{NH}_{2}$ ○ $\mathrm{C}_{2} \mathrm{H}_{5} \mathrm{NH}_{2}$. Para cada uma das ominas, detectam-se o fon molecular de massa $\mathrm{M}$, assim como os lons de masse $M-1$, e $1+1$. Os lons protonados das aninas são fons sccundérios, de acôrdo dos argumentos ilustrados nos parágrafos anteriores. O moconismo de formasão dêstes íns protonados não \& de interêsse imediato. Porém, independentemente de origem dêsses lons, as formas protoncdas estarão Iigadas pela reação quínica (3-2), se houver colisões tentondo estabelecer equilíbrio quírico. 


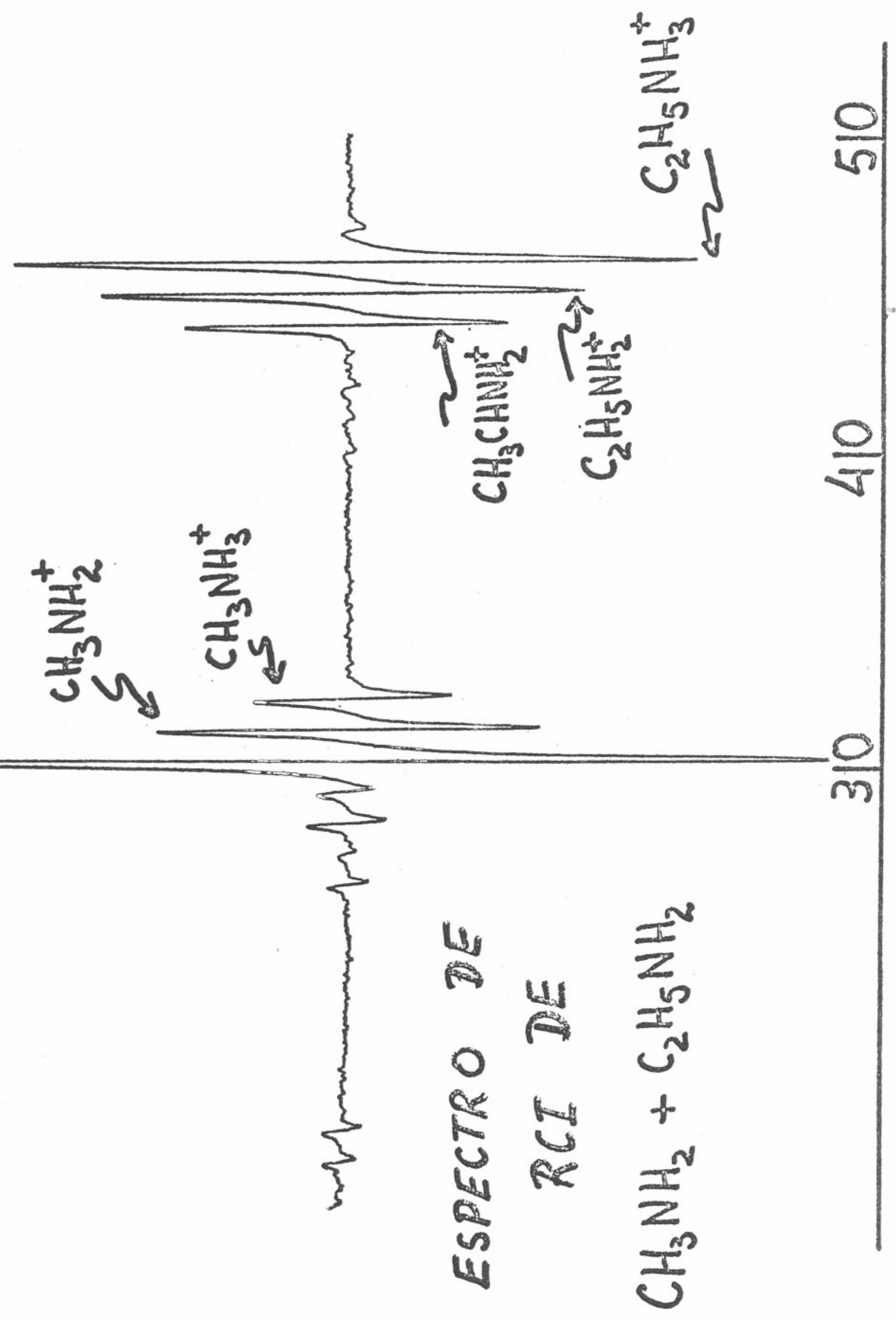

Iuvil 3-2 


$$
\mathrm{CH}_{3} \mathrm{NH}_{3}^{+}+\mathrm{C}_{2} \mathrm{H}_{5} \mathrm{IIH}_{2} \longleftrightarrow \mathrm{CH}_{3} \mathrm{IHH}_{2}+\mathrm{C}_{2} \mathrm{H}_{5} \mathrm{HII}_{3}^{+}
$$

A reação qufmics (3-2) ñ̃o protondo isolor ôsto único processo dos lons protonados; s6 estebelece und possivel comunicação ontre as duas mincs protonades através de tronsfurêncio. do un proton. A direção preferencial do equilíbrio não é ospecificada porque desconhocon-se o priori os dados temodinâmicos da roạ̃ão, c porque o espectro não rofleto nocesshricmento uma condição de equilíbrio ostetístico entro reogentes - produtos para a recsão postulada. Fubora as constontes do velocidade perc. êste tipo de reção sejon nuito ultas, o nínero do colisões as pressões estudades ( $10^{-5}$ torr.) não coranto essa condiç̃̃o de oquilfbrio.

A ocorrência da reação (3-2) na cela pode sor testado. por meio do metodo de ressonência dupla. 0 ion $\mathrm{C}_{2} \mathrm{H}_{5} \mathrm{IHH}_{3}{ }^{+}$esteré cm ressonência quando o oscilcdor morgincl fôr fixado numa frequência $\omega_{1} \neq 153,6 \mathrm{kHz}$ c 0 compo magnético on 4600 gauss. Se concomitante com esta observação, o segundo oscilcdor fôr varrido na região da Irequêncic ciclotrônica de $\mathrm{CH}_{3} \mathrm{HH}_{3}{ }^{+}$para um campo magnático do 4600 gauss,

$$
\omega_{2}=\frac{M\left(\mathrm{C}_{2} \mathrm{H} 5^{\mathrm{MH}} 3^{+}\right)}{M\left(\mathrm{CH}_{3} \mathrm{HH}_{3}{ }^{+}\right)} \omega_{1}=220,5 \mathrm{kHz}
$$

serd posslvel doterminar de acôrdo com os princlpios estabelecidos no segundo capftulo, se os dois fons protoncdos estão ligeados ontre si por uma reação quínica.

No figura 3-3, o espectro de ressonôncic dupla é ilusibla do para o ín $\mathrm{C}_{2} \mathrm{H}_{5} \mathrm{NHH}_{3}^{+}$. iste espectro foi obtido modulando a anplitude do segundo campo de codiofrequencia, $\mathbb{E}\left(\omega_{;}\right), \infty$ 
ESPICTRO DE RESSONANCIA DUPLA DO ION $\mathrm{C}_{2} \mathrm{H}_{5} \mathrm{NH}_{3}^{+}$
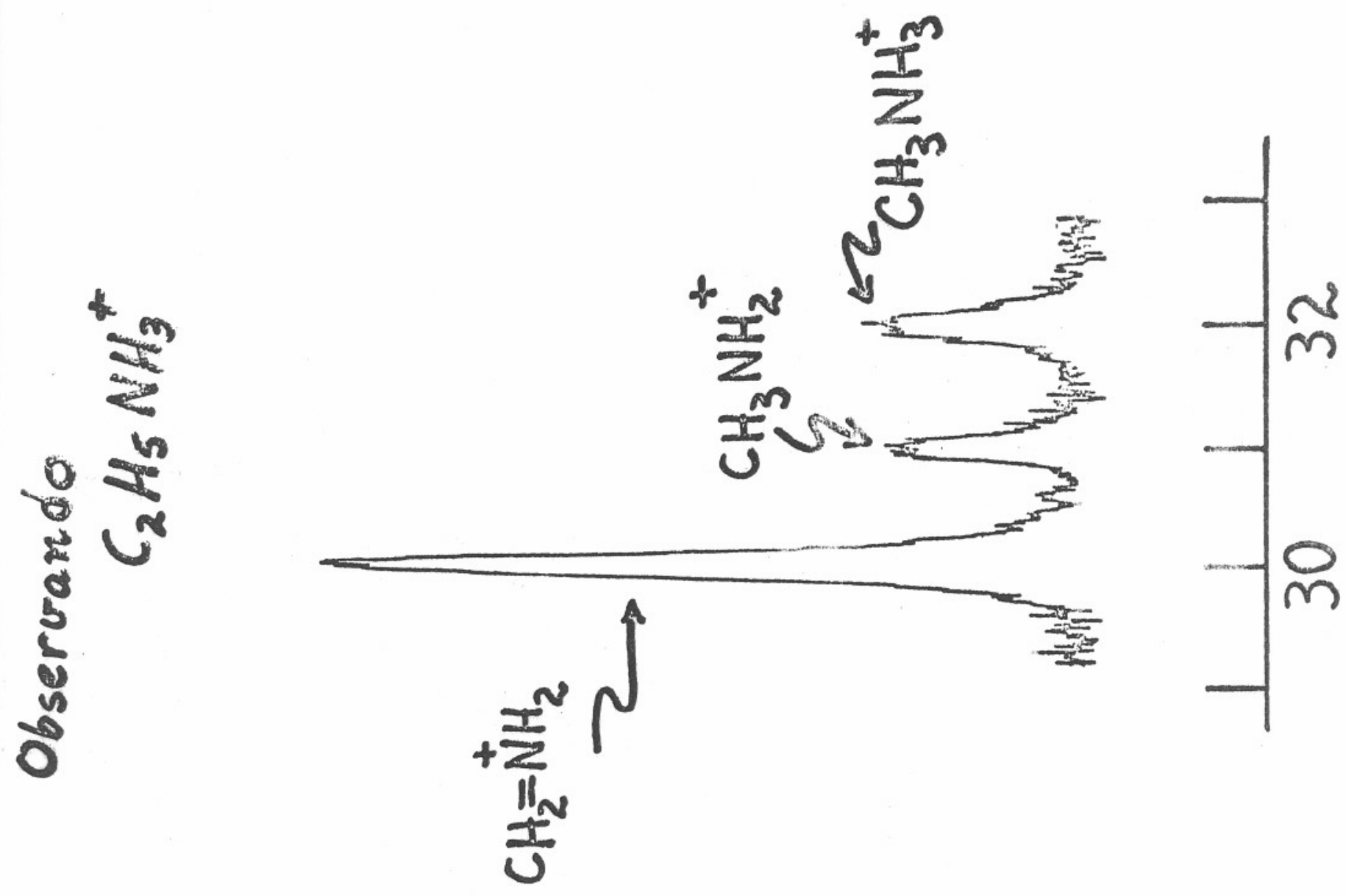

FIGURA $\quad 3-3$ 
mosmo tompo que ôlo é vorrido na rogiõo dos íons dorivados de $\mathrm{CH}_{3} \mathrm{NH}_{2}$. îsto espectro revola ofotivanente aue $\mathrm{CH}_{3} \mathrm{NH}_{3}^{+}$osté Iigcdo a $\mathrm{C}_{2} \mathrm{H}_{5} \mathrm{NH}_{3}{ }^{+}$atrovés da roaça postulada. Da mosma manoiro, - ospectro indice de que os lons $\mathrm{CH}_{2} \mathrm{IH}_{2}{ }^{+}$o $\mathrm{CH}_{3} \mathrm{NH}_{2}^{+}$contribuom tambóm pare fomação de $\mathrm{C}_{2} \mathrm{H}_{5} \mathrm{MH}_{3}{ }^{+}$atrovés do roaçõos qui-micas.

Astes resultados garanton ontão que as squaçõos de movimento de $\mathrm{CH}_{3} \mathrm{NH}_{3}{ }^{+} \mathrm{e} \mathrm{C}_{2} \mathrm{H}_{5} \mathrm{ITH}_{3}{ }^{+}$estão acopladcs. Alcm disso s de interêssc conhocer os dados temodinêmicos dosto reaç̃o, je que ola reprosonta a competição do duas amines para com o proton, e portanto capaz de estabelecer a basiciado relotive dos duas aninos om discussão.

Os espectros de ressonância simples en funçõo da pressão constituiriom en princlpio as bascs de um método qualitativo para obter informoção sôbre a bosicidade rolativa das duas minas. A intensidade dos lons corrospondentes indiccria nêsse caso qual dêsses lons fornados f favorocido temodinânicamonte. Asto tipo de ergumento $s$ semelhante do uscdo por Munson. 28 Porón, esta linha do pensamento pressupõe misturas gasosas equimolares, ausência do bomboio preforcneiol na cela, o equiIfbrio estatístico intemo. Hstas suposiçoes podom ser perticuIcmonte criticas no caso do reaşos nes quais a cnergia livro da reação é muito pequenc, como no caso considerado.

A intorprotação dos dados temodinâmicos da reação (3-3), assim como também o de outras somelhantes, é bascada noste trabalho, no sinal do espectro de ressonância dupla. Porém, êsses argumentos dependern de certos aspectos das reacões entre íns e moleculas neutras na fase gasosa. 
III.3 Consideracões sôbre as reacões entre fons e moléculas na fose gasosa

1 reação (3--2) constitui un caso típico de uña reação entre fon e molécula neutra na fase gasosa. Nste tipo de reacões, cujos estudos foran iniciados na década de 1950, caraterizam-se por urna constante de velocidade muito alte (de orden de $10^{11}$ mol $1^{-1} 1$ seg $^{-1}$ ), e a cusência de uma energia de ativacão para o processo cinetico. $31-34$

Os aspectos puranente dinâmicos destas reações podem ser interpretodos com o jé clássico modêlo de Gioumousis e Stevenson. ${ }^{35}$ Neste modêlo, a reação de $\mathrm{CH}_{3} 3_{3}{ }^{+}$e $\mathrm{C}_{2} \mathrm{H}_{5} 5^{\mathrm{MII}} 2$ é visualizada en têrmos das trajetórias clássicas para colisão bimolecular sob a influência de un potencial de atração. Na figura 3-4, a molécula de $\mathrm{C}_{2} \mathrm{H}_{5} \mathrm{NH}_{2}$ é identificada como um ponto fixo, $\mathrm{B}$, enquanto que o lon, $\mathrm{A}^{+}$, oproxima-se corn ume velocidade relativa $\mathrm{V}$ e com um "parêmetro de impacto" inicial b.

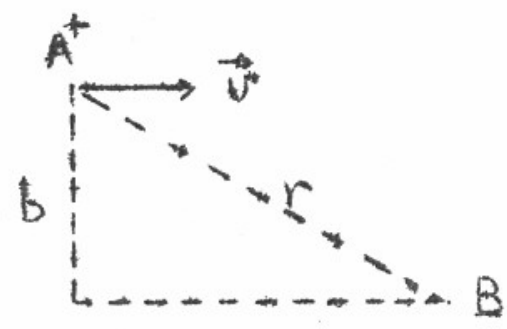

Figura $3-4$

- potencial de interação introduzido no problema é aquêle entre urna carga a e urna molécula polarizável B.

$$
V(r)=-\frac{\alpha q^{2}}{2 r^{4}}
$$

$\alpha$-.... olerizabilidade de $B$ 
B̂ste simples potencial não leve en conte a possível existôncia d.e un momento dipoler permonente no molecula, ou os têrmos do potencial de Iennard-Jones. A trajetbria de $\mathrm{A}^{+}$será alterada pelo potencial de atração, de tol naneira que para parênetros de inpectos iniciais menores do que un valor critico, $b_{0}$, - Ion $\mathrm{A}^{+}$entraré em brbita espiral de raio decrescente 0.0 redor de B. A trajetório do $A^{+}$chegoré a possar pelo centro de $B$, se um potencial de repulsão (do tipo $r^{-12}$ por exomplo) não fôr adicionado 20 problema. Êste valor crettico de $\underline{b}$ pode ser determinado, analisando detalhadarnente o problema necênico. 36

$$
b_{c}=\left(\frac{4 q^{2} \alpha}{\mu v^{2}}\right)^{1 / 4}
$$

( $\mu$-..- massa reduzida dos reagentes)

Se cada colisão caraterizada por un perâmetro de inpacto nenor que $b_{0}$ é capaz de produzir una reação quínica, a seção de choque para o processo será

$$
\sigma(v)=\pi h_{0}^{2}-\frac{2 \pi}{\omega}\left(\frac{q^{2} \alpha}{\mu}\right)^{1 / 2}
$$

(Esta formula será vélida so para reações exotémicos onde a energia de ativecão é considerada nula. Para procossos endotémicos, a propria endotemicidade representoric a onergic de ativação).

Da1, calcula-se a constante do velocidade da reasão química por meio da equação (3-6), (ver referência 37)

$$
k=v \sigma
$$


Para o modêlo on questão,

$$
k=2 \pi q\left(\frac{\alpha}{\mu}\right)^{1 / 2}
$$

istes conceitos sinples produzen resultados qualitativos altamente satisfatbrios para reaçõos exotemicas. Assim, a equação (3-7) prediz corretomente a ordem de frondeza da constante de velocidade, e domonstra que $\mathrm{k}$ independe da temperatura. Imentavelmente, a constante de velocidade do modêlo de Giounousis e Stevenson independe da velocidode relativa das partf́culas, em desacôrdo com resultados experimentais. Ume interpretação quantitativa dos resultados experimenteis en função desta teoria nern sempre é possivel, devido ds simplificações conceituais do modôlo, e sobretudo do potencial de interação. As linitações dêste tratamento pera os detaihes obtidos pelos métodos uxperimentais mais caprichados, assin como estudos sôbre a variação da constante $\underline{k}$ com a chorgia translacional fazen parte da excolente monogrofia publicada pela American Chemical Society. 38

Conclui-se destas considerações, que pera fins préticos e como boa aproximação, as secções de choque colculcdas para colisões "Intimas" (acuelas con $b<b_{0}$ ) correspondon is seções de choque para roções exotémicas.

\section{III.4 Significado cinético da experiência de ressonência}

\section{ciclotrônica}

Beauchomp o Butrill 39 analisarcm a cinética de um Ion primério para produzir un fon secundério sob as condicõos de observação do espectro de rossonância ciclotrônica simples 
- dupla. O fato da roação (3-2) onvolver fons socundírios como reagentes so altora a nomoncl tura do problona.

Os lons $\mathrm{C}_{2} \mathrm{H}_{5} \mathrm{IHH}_{3}{ }^{+}$detectodos pelo oscildor merginal sc̃o devidos a uma série de processos guímicos responś́veis pela sua fomação. Prosume-se que todos âstes procossos ocorrem a nivel da onergia tómica, mas as restricõos sôbro esta hipótese serão discutidas mois adiante. Quando um dos Ions responsáveis pela formação de $\mathrm{C}_{2} \mathrm{H}_{5} \mathrm{HH}_{3}{ }^{+}$e ccelercdo por un segundo campo de radiorrequência, $\mathrm{E}_{2}\left(\omega_{z}\right)$ (ñ̃o confundir com - $\mathrm{P}_{2}$ do prineiro capitulo), equivale a dizer quo o lon incidente, $\mathrm{CH}_{3} \mathrm{NH}_{3}^{+}$no caso da reação (3-2), roogo com $\mathrm{C}_{2} \mathrm{H}_{5} \mathrm{MH}_{2}$ com una onegia cinética naior do que a onergia têmica. Assin, a energia cinstica translacional do fon reagento na experiencia de ressonância dupla, linitada por colisões olḱsticas que dissipam essa energia em excesso (ver II.3e o (2-10)), pode ser derivada a partir da express̃̃o de $A\left(w_{z}\right)$.

4

$$
E_{\mathrm{CH}_{3} N H_{3}^{+}}=\frac{3}{2} k T+\left[(m+M) A\left(\omega \omega_{2}^{*}\right) / 2 \nu m n^{+}\right]
$$

m --- massa de $\mathrm{CH}_{3} \mathrm{IHH}_{3}^{+}$

Ni --- massa da molécula responsávol pela colisão dissiponte.

Quando $\omega_{2}=\omega\left(\mathrm{CH}_{3} \mathrm{NII}_{3}^{+}\right)$

$$
E_{\mathrm{CH}_{3} \mathrm{NH}_{3}^{*}}=\frac{3}{2} R T+\left[q^{2} E_{2^{2}}^{2}(m+M) / 8 v^{2} m^{2}\right]
$$

Consequentencnte, se $\omega_{2}$ coincidir conl co frequôncia ciclotrônica do íon $\mathrm{CH}_{3} \mathrm{NHE}_{3}{ }^{+}$, o processo cinético de formoção de $\mathrm{C}_{2} \mathrm{H}_{5} 5^{\mathrm{HEI}}{ }_{3}^{+}$a partir dos reagentes já ruencionedos soŕb altorado, se o processo depender da onergio translacional do fon reogente. Como foi mencionado no item III.3, o a despoito do 
que a teoria de Giournousis o Stevenson prediz, cs constantes de velocidade desto,s reçõos dependon de onergie translccionol.

A. experiência de ressonêncic dupla, cujos rosultados ospecificos forom mostrados na figura $3-3$, \& roolizada modulando a. $38 \mathrm{~Hz}$ a voltagem de salda do oscilador de erequencia $\omega_{2}$, ontre $\mathbb{E}=0$ e $\mathbb{P}=\mathbb{E}_{2}$. Isto implica cin que nume metede do ciclo de modulaç̃o, alguns lons $\mathrm{C}_{2} \mathrm{H}_{5} \mathrm{HH}_{3}{ }^{+}$estão sondo fomados por por $\mathrm{CH}_{3} \mathrm{NH}_{3}{ }^{+}$com onorgia translacional igual a $3 / 2 \mathrm{kT}$, enquento que na outra metade do ciclo astão sendo formados por fons con energia cinética dada pela equaçó (3-9). (I mbror que $\forall \gg 35 \mathrm{~Hz}$ ). Se o detector de fese fôr ajustedo com rolaç̃o ̀े frequencia de nodulç̧̃o do oscilador $\omega_{2}$, o sinal registrado serć do intensidade $\Delta I$, definido om unidodes croitrórics pola equeção (3--10).

$$
\Delta I=I_{R D}-I_{R S}
$$

$I_{R S}$-- intensidade da absorção de onergic por perte dos fons $\mathrm{C}_{2} \mathrm{H}_{5} 5^{\mathrm{NH}_{3}}{ }^{+}$na experiência de ressonencia simplos, quendo os Lons reagentes estão corcterizados por uma onergio tómica.

$I_{R D}$-- intensidado da absorção de cnergia por purte dos fons

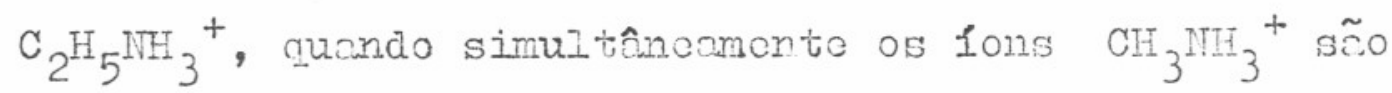
acolorados o possuen encrgia tronslecionol acima da energia térmica.

Estes intensidades individuais sc̃o dirotomonto proporcionais a número do fons que ebsorve onergic do oscilcdor primório. Âste número de fons presente no cola deponde de una séric de processos cinéticos e suas constantes de velocidode correspondentes. Como a diferença de intensidade representeda 
pela equaç̃o (3-10), reflete s6 a mudunça do processo (3-2) e de sua constante de velocidade, obtén-se

$$
I \propto\left(k_{\mathrm{RD}}-k_{\mathrm{RS}}\right)
$$

Como as experiências de ressonencia dupla sc̃o realizadas a compos elétricos fracos ( $\mathrm{E}_{2}$ móxino $\sim 0,1 \mathrm{~V} / \mathrm{cm}$ ), a energia cinética de $\mathrm{CH}_{3} \mathrm{NH}_{3}{ }^{+}$será aponas ligeirmente cumentada com relação a $3 / 2 \mathrm{kT}$. Quando $\mathrm{E}_{2}$ fôr suficientomente fraco de tal maneira que

$$
\frac{q^{2} E_{2}^{2}(m+M)}{\& v^{2} m^{2}} \ll \frac{3}{2} k T
$$

a constante de velocidade, $k_{R D}$, poderé ser expresse por une serie de Taylor.

$$
k_{R D}=k_{\text {TÉRMiCO }}+\frac{d k}{d E_{\mathrm{CH}_{3} \mathrm{NH}_{3}^{+}}}\left(E_{\mathrm{CH}_{3} \mathrm{NH}_{3}^{+}}-\frac{3}{2} k_{T} T\right)
$$

( $\mathrm{E}_{\mathrm{CH}} \mathrm{NH}_{3}{ }^{+}$-- energia cinstica translacional do fon $\mathrm{CH}_{3} \mathrm{NH}_{3}{ }^{+}$) Ienbrondo que $k_{\text {térmico }}=k_{\text {RS }}$, a conclusĩo final é que sob a.s condições representadas pela equaç̃o (3-12), tem-se

$$
\Delta I \propto \frac{d k}{d E_{\text {tians }}}
$$

Isto implica on que a exporiêncio de ressonencia dupla pode ser utilizada para obter informações sôbre a variação da constante de velocidade en função da energia translacional do reagente. 
III.5 Uso da voriaça da constonte de velocidade com energie tronslacional do fon reagente pore doterninor a ofinidade protônica das aminos

Pelos motivos apontados no ftem III.3, o salientando - pequeno $\Delta H$ esperado pera reações do tipo (3-2), a comperaça da intensidade dos picos dos 1ons protoncdos no ospectro do mistura de $\mathrm{CH}_{3} \mathrm{IH}_{2}$ e $\mathrm{C}_{2} \mathrm{H}_{5} \mathrm{HH}_{2}$ não revela de maneira brvia a sua cefinidade protônica relotiva.

Intretanto, as conclusões resumidas na equação (3-14) podem ser aproveitadas de maneira inesperada como urn teste dos propriedades termodinâmicas. A relaçõo entre (dk/dت trons e $\triangle \mathrm{AH}$ de uma reação entre íons e moléculas na fese gasose foi estabelecida por Beauchanp e Butrill, 39 e confimada polos resultados recontes de Bowers, IIlemon c King. ${ }^{18}$ Os princípios estabelecidos nôsses trabolhos indicam: (a) qunndo - $\Delta I$ observado experimentalmente é uma grendeza negativa, a reação testada deve ser exotemica;(b) se o $\Delta I$ obtido por ressonûncia dupla fôr positivo, a reção pode ser exotémico ui cndotérmica. Estc relnção cmpírica ontre as propriedades termodinâraicas de reação e a voriação de constonte do velocidede com a onergic translacional esté vinculade de cusoncie do onergia de ativação para êstos processos, e ̀̀s peculiaridades das reações entre 1ons e moléculas. De forma geral, estas observaçós sustentan as conclusões mais importantes de teoria de Iight, ${ }^{40}$ que considera colisões "ergódicos" e os possíveis cancis de decomposição de un complexo de colisz̃o.

Mais especyficmente, tem-se obscrvado que para reações de simples transferência de prótons ontre duas entidedes moleculares, as reaçõos oxotérmicas estão senpre caraterizadas 
por un $\Delta I$ negativo.

Dada ontão uma reaç̃o groral do tipo

$$
\mathrm{RNH}_{3}++\mathrm{R}^{\prime} \mathrm{NH}_{2} \rightleftarrows \mathrm{RNH}_{2}+\mathrm{R}^{\prime} \mathrm{NH}_{3}^{+}
$$

onde as afinidodes protônicas das duas anines, $\mathrm{RIH}_{2}$ e $\mathrm{R}^{\prime} \mathrm{NH}_{2}$, são desconhecidas, mas presumlvelmonte semelhantes devido לे observação dos dois picos protonados no espectro de ressonincia simples, a temoqulnica da reação pode ser inforida a partir das seguintes experiências:

(A) Os fons de $\mathrm{R}^{\prime} \mathrm{NH}_{3}{ }^{+}$são detectados (ou observados) atraves do oscilador marginal, fixo na frequência ciclotrônica dos mosmos, encuanto que o segundo oscilador de frequência $\omega_{2}$, igual a frequência ciclotrônica de $\mathrm{RNH}_{3}{ }^{+}$, e pulsado da manoiro indicada no ftem III.4. Da1, $0 \Delta I$ pode sor observado cono uma grondeza positiva ou negativa.

(B) 0 processo \& agora invertido, observando-se $\mathrm{RNH}_{3}{ }^{+}$ enquanto a cnergie translecional de $\mathrm{R}_{\mathrm{NH}}{ }_{3}^{+}$e pulscda ctravés do segundo oscilador. O A I rogistrodo indica a caraterística tomodinâmica da reaçõo (3-15) na direçõo de diroita para esquerda.

Os resultados destes experiências podem ser enquadrados dentro das considereçoos gerais para reaçõs de tronsferôncia. de protons entre molsculas polietômicas no fase gasosa.

1) Se a roação (3-15) estudade no sentido de escuerda para direita (oxperiôncia $A, c-. . \rightarrow$ d) produz un $\Delta$ I neğtivo, existem duas ossibilidades para a experiência $B$.

(a) $\Delta I($ d $\rightarrow$ e) e neğativo. Nôste caso, a de ccôra con as regras gerais que form especificadas, a reaçõo (3-15) seria. 
aparontononte exotérmica nos dois sentidos. Fusiconente, este interpreteção implica en que a reação é temonoutral, já que - pequeno excesso de chergie comunicado do fon reagente pelo oscilador de rossonancic duplo \& suficiente pora produzir uma reação exotémica. Sob estas condições, conclui-se que

$$
\text { A.P. }\left(\mathrm{RNH}_{2}\right)=\text { A.P. }\left(\mathrm{R}^{\prime} \mathrm{NH}_{2}\right)
$$

(b) $\Delta I(d \rightarrow$ c) e positivo. Hsta observação indica a ondotermicidade de reç̧̃o de direita para esquerde. A conclusc̃o \& sbria,

$$
\text { A.P. }\left(R \cdot \mathrm{NH}_{2}\right)>\text { A.P. }\left(\mathrm{RNH}_{2}\right)
$$

2) Se a reação (3-15) no sontido de escuerda paro direite produz un $\Delta I$ positivo no espectro de ressonência dupla, a experiencia $B$ so apresonta ume possibilidade: $\Delta I(d \rightarrow 0)$ \& negativo. Consequentenente, as indicações aquí ossinalom

$$
\text { A.P. }\left(\mathrm{RNH}_{2}\right)>\text { A.P. }\left(\mathrm{R}^{\prime} \mathrm{NH}_{2}\right)
$$

A segunda possibilidade, ou soja $\Delta I(d \rightarrow 0)$ positivo, nunca foi obscrvada, e sua ocorrencie inplicaria ne não validez dos argumentos ats aque expressos.

Estes rosultados que permitem a inforôncio de dados termodinênicos constituom un mótodo muito vontajoso e sonsível para detominer as afinidades protônicas relativos. Porén, convóm insistir on que os peculiaridades nas quais ostes resultados estão bascodos, so são aplicóveis pare êste tipo de reações. 
Os conceitos elaborados neste terceiro capitulo foram aplicados no estudo das seguintes aminas: $\mathrm{CH}_{3} \mathrm{HI}_{2}, \mathrm{C}_{2} \mathrm{H}_{5} \mathrm{HH}_{2}$, $\mathrm{CH}_{3} \mathrm{CH}_{2} \mathrm{CH}_{2} \mathrm{NH}_{2},\left(\mathrm{CH}_{3}\right)_{2} \mathrm{CHNH}_{2},\left(\mathrm{CH}_{3}\right)_{3} \mathrm{CHH}_{2},\left(\mathrm{CH}_{3}\right)_{3} \mathrm{CCH}_{2} \mathrm{NH}_{2}$, $\left(\mathrm{CH}_{3}\right)_{2} \mathrm{NH},\left(\mathrm{CH}_{3}\right)_{3} \mathrm{NT},\left(\mathrm{C}_{2} \mathrm{H}_{5}\right)_{2} \mathrm{MH},\left(\mathrm{C}_{2} \mathrm{H}_{5}\right)_{3} \mathrm{~N}$. As crincs gesosas a temperatura anbiente foram obtidas en cilindros da Iratheson Scientific Company, enquonto que as restantes form adquiridas de. Mathoson, Coleman e Bell. Atraves dos espectros de ressonância simples demonstrou-so un alto toor de pureza nestes conpostos, e, consogtentemonte não foi necesserio purificé-los.

Os espectros do ressonancia simples de code ume destos aninas form cnalisados a ume onergia cletrônico nominal do $16 \mathrm{cV}$. Estes espectros serviram pare identificar os picos mois importantos produzidos polos aminas, como jó tôno indicado na parte III.2, o sobretudo para doteminar os pressões mais adequadas para a observação dos lons protonados de masse $1 \Gamma+1$. (Ir represente o pêso moleculor de anina). Pressõos de $10^{-5}$ torr foran dotominadas coro as mais conveniontes pare êstos ostudos. Os espectros forem obtidos com o espectrômetro ¿ tomperaturo. ambiento, nume scla regulada a $20 \pm 2{ }^{\circ} \mathrm{C}$.

A soguir, misturas coroximodomonte equimoloros do duas aninos eron preparacias numo linha de vócuo convencional, pera - estudo da reação (3-15) correspondente por ressonâncie duple. Uma vêz detominadas as condiçõos ideais para a observaçño dos Ions protonados das duas aninas en comparaça, os espectros de ressonência dupla cram obtidos do acôrdo con o esquome desonhado na parte III.5 - Os espectros de ressonência dupla orn obtidos c. una encrgia eletrônica nominal de $16 \mathrm{cV}$, e tombóm è onorgis cletrônica mínima necossciria para a observaça dos fons proto- 
nedos $(10010,5 \mathrm{eV})$. Os aspectros de ressonfncie duple obtidos a energia eletrônice minime eron de intensidace muito frcca, mos nunca foi observado uno mudança no sincl lfobbrico de $\triangle I$ entre as duas experioncias.

A intorprotaça de $\Delta I$ como ume grandoz positive ou negativa depende do contrôle de fase do detector o anplificador, a por tonto foi necosserio resolver esse crbitrariodcdo ontes das experiôncias. Dois métodos form usados porn calibror o detector de maneira $\mathrm{c}$ seber quando $\Delta I$ roprosutave un corbocimo, ou un decrescino com relação ì intensidade do ospectro de ressonincia simples:

1) 0 espectro de ressonência simples ore obsorvado modulando - campo magnetico enquanto que o segundo oscilador ors usado on fome continua ne froquência ciclotrônice co lon rocgento. in picos muito intonsos, era posslvel observar um pequono acréscino, ou decréscimo, ne. primeira derivade da linha de absorção. 2) Un criterio nais simplos consistia on colibrer o dotoctor usculo como padrão a reaşão

$$
\left(\mathrm{CH}_{3}\right)_{2} \mathrm{CHOH}_{2}++\left(\mathrm{CH}_{3}\right)_{3} \mathrm{COH} \rightarrow\left(\mathrm{CH}_{3}\right)_{2} \mathrm{CHOH}+\left(\mathrm{CH}_{3}\right)_{3} \mathrm{COH}_{2}^{+}
$$

a cuel produz un $\Delta I$ nogetivo. 16

O contrôle de fose no detector foi estabelecido de tal moneira aue os espectros de ressonência dupla roprosontem un

$\triangle I$ negativo quando os picos soben com rolcešo a Iimho base do espectro, e viceversa.

Un rosuno das misturas analisadas \& aprosentado a seguir com mais detalhes. 
$\mathrm{CH}_{3} \mathrm{IHH}_{2}+\mathrm{C}_{2} \mathrm{H}_{5} \mathrm{HH}_{2}$ : As carcterísticas do espectro do rossonêneic simples e duple je foran pomonorizodas nas fisuras $3-2$ e 3-3. o ospectro de ressonsncie dupla obticto quando o ospectrómetro este sob condições de ressonência parc o lon $\mathrm{C}_{2} \mathrm{H}_{5} 5^{\mathrm{NH}}{ }^{+}$dononstra que a resção,

$$
\mathrm{CH}_{3} \mathrm{NH}_{3}{ }^{+}+\mathrm{C}_{2} \mathrm{H}_{5} \mathrm{IHI}_{2} \rightarrow \mathrm{CH}_{3} \mathrm{IHH}_{2}+\mathrm{C}_{2} \mathrm{HH}_{5} \mathrm{MH}_{3}{ }^{+}
$$

é exotémica devido ao $\triangle I$ negetivo observado. A experiôncic oposta, aquela na qual o lon $\mathrm{CH}_{3} \mathrm{NH}_{3}{ }^{+}$\& observado, enquanto que $\mathrm{C}_{2} \mathrm{H}_{5}{ }^{\mathrm{IH}}{ }^{+}$e irrodicdo com o secundo oscilcdor, nõo produziu un espectro de ressonância dupla.

$\mathrm{C}_{2} \mathrm{H}_{5} \mathrm{NH}_{2}+\mathrm{CH}_{3} \mathrm{CH}_{2} \mathrm{CH}_{2} \mathrm{NH}_{2}$ (Vor figura 3-5). Os picos protoncios de intchêsse corresponaen c. $\mathrm{C}_{2} \mathrm{H}_{5} \mathrm{NH}_{3}^{+}(\mathrm{II}=46), \quad \mathrm{C}_{3} \mathrm{H}_{7} \mathrm{HH}_{3}^{+}$ $(M=60)$. As experiôncias de ressonêncie dupla indicon cue a reaçõo

$$
\mathrm{C}_{2} \mathrm{H}_{5} \mathrm{NH}_{3}^{+}+\mathrm{CH}_{3} \mathrm{CH}_{2} \mathrm{CH}_{2} \mathrm{INH}_{2} \longrightarrow \mathrm{C}_{2} \mathrm{H}_{5} \mathrm{NH}_{2}+\mathrm{C}_{3} \mathrm{H}_{7} \mathrm{IH}_{3}^{+}
$$

\& cxotermica, enquanto que a roaçõo invorso rovola as coratoristicas de uno reação endotormica. (Os espectros de figure 3-5 mostram un dotalho muito intoresscnte: o sinal observado pare. reações ndotomicas sb cparce sindtrico a canpos elbtricos de irrediação muito fracos. Uma cxplicação qualitativa dôsto ofeito, o seu significcdo flsjco, é discutido por Bowers ${ }^{18}$ ). $\mathrm{C}_{2} \mathrm{H}_{5} \mathrm{IH}_{2}+\left(\mathrm{CH}_{3}\right)_{2} \mathrm{CHNH}_{2}:$ A experiêncid de ressonincie duple pern esta misture demonstrou cuo a reção,

$$
\mathrm{C}_{2} \mathrm{H}_{5} \mathrm{NH}_{3}^{+}+\left(\mathrm{CH}_{3}\right)_{2} \mathrm{CHNH}_{2} \longrightarrow \mathrm{C}_{2} \mathrm{H}_{5} \mathrm{MH}_{2}+\left(\mathrm{CH}_{3}\right)_{2} \mathrm{CHNH}_{3}^{+}
$$

e oxotémica. A despoito de muitos tontativas, a raaš̃o invorsa. não foi detectada por rossonência duple. 

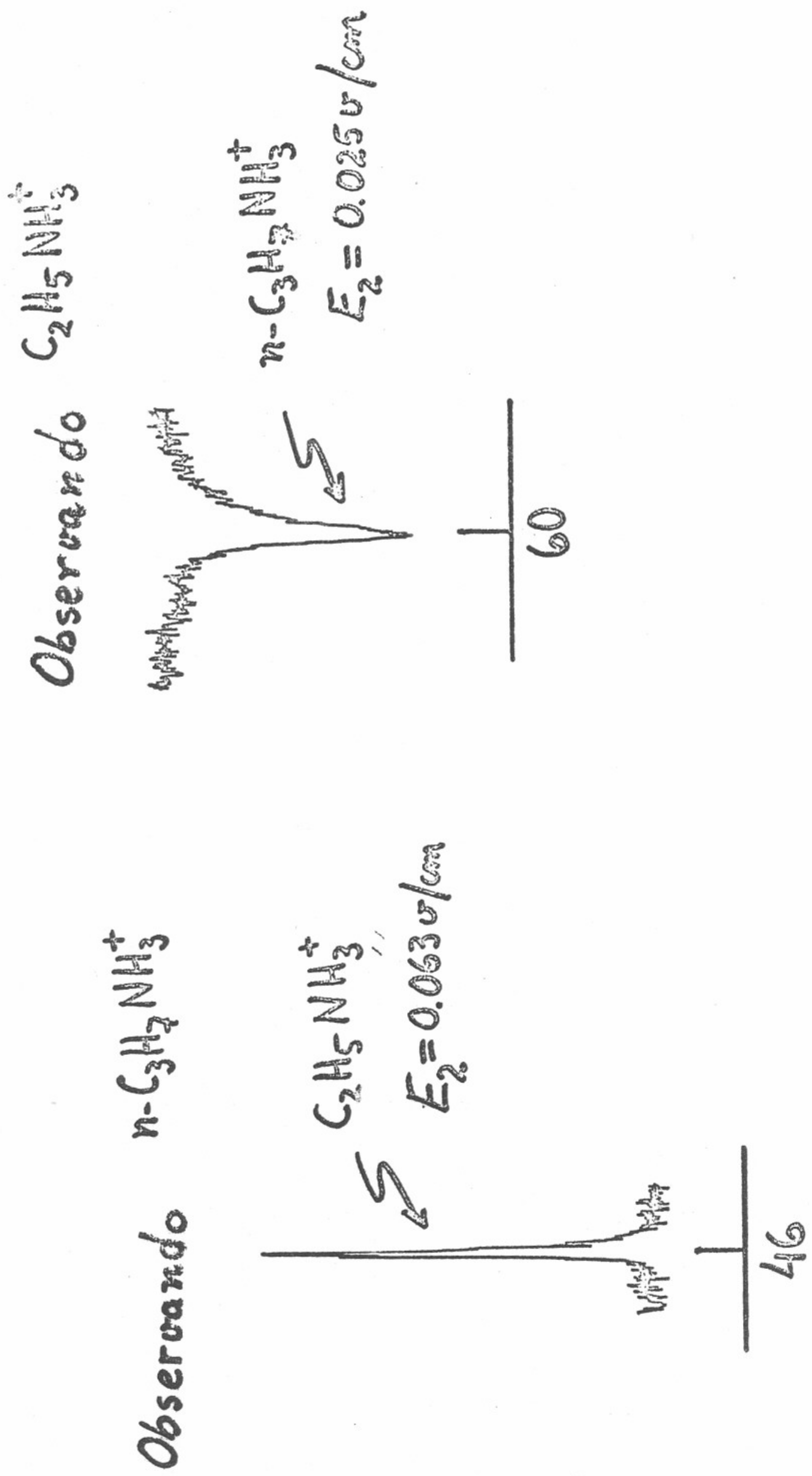

FIGURA $\quad 3-5$

Espectros de ressonência dupla usados pra deteminar a bssicidaae relativa da etil amina e a propil anina. O valor de $\mathrm{E}_{2}$ se refere a amplitude da radiofrequência do segundo. ascilndor. 
$\mathrm{CH}_{3} \mathrm{CH}_{2} \mathrm{CH}_{2} \mathrm{HH}_{2}+\left(\mathrm{CH}_{3}\right)_{3} \mathrm{CNH}_{2}$ : Os resultados obtidos por ressonenncio dupla pemiten concluir que a reação,

$$
\mathrm{CH}_{3} \mathrm{CH}_{2} \mathrm{CH}_{2} \mathrm{NH}_{3}^{+}+\left(\mathrm{CH}_{3}\right)_{3} \mathrm{CNH}_{2} \longrightarrow \mathrm{CH}_{3} \mathrm{CH}_{2} \mathrm{CH}_{2} \mathrm{NH}_{2}+\left(\mathrm{CH}_{3}\right)_{3} \mathrm{CNH}_{3}^{+}
$$

s exotemnica. A roação inversa produz um AI muito fraco e positivo, indicondo a endotemicidcde dessa reação.

$\left(\mathrm{CH}_{3}\right)_{2} \mathrm{CHNH}_{2}+\left(\mathrm{CH}_{3}\right)_{3} \mathrm{CNH}_{2}: \mathrm{A}$ intorpretação dos resultedos destes experiôncias é semelhonte d da experiêncis cnterior, indicando que a afinidade protônice de $\left(\mathrm{CH}_{3}\right)_{3} \mathrm{ClNH}_{2}$ é maior do que a A.P. de $\left(\mathrm{CH}_{3}\right)_{2} \mathrm{CHNH}_{2} \cdot 0$ sinal de reação endotêmice pare ôste caso \& bem mais pronunciado do que no caso anterior.

$\left(\mathrm{CH}_{3}\right)_{3} \mathrm{CHH}_{2}+\left(\mathrm{CH}_{3}\right)_{3} \mathrm{C}-\mathrm{CH}_{2} \mathrm{NH}_{2}:$ (Ver Pigure 3-6). Esto misture, de grande interosse devido aos rosultodos obtidos por Bloir o Brauman pera alcóxicos, ${ }^{24}$ rovele que

$$
\left(\mathrm{CH}_{3}\right)_{3} \mathrm{CNH}_{2}+\left(\mathrm{CH}_{3}\right)_{3} \mathrm{C}-\mathrm{CH}_{2} \mathrm{NH}_{3}^{+} \rightarrow\left(\mathrm{CH}_{3}\right)_{3} \mathrm{CNH}_{3}^{+}+\left(\mathrm{CH}_{3}\right)_{3} \mathrm{C}^{+} \mathrm{CH}_{2} \mathrm{NH}_{2}
$$

\& um procosso exotemico, onquanto que a reação invorso de protonação revcla as carcterísticas de ume reação cndotóruica.

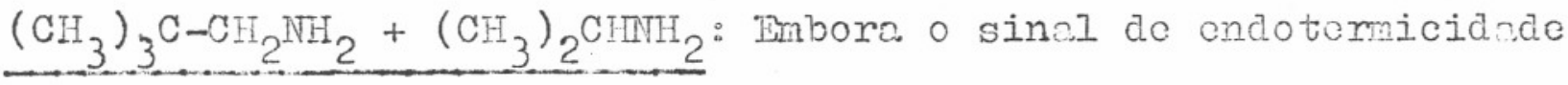
seja muito froco perc êsto coso, conclui-se dos experiencias de ressonincia dupla que

$$
\text { A.P. de }\left(\mathrm{CH}_{3}\right)_{3} \mathrm{C}-\mathrm{CH}_{2} \mathrm{NH}_{2}>\text { A.P. de }\left(\mathrm{CH}_{3}\right)_{2} \mathrm{CHNH}_{2}
$$

$\left(\mathrm{C}_{2} \mathrm{H}_{5}\right)_{2}{ }^{\mathrm{NH}}+\mathrm{C}_{2} \mathrm{H}_{5} \mathrm{NH}_{2}: \mathrm{A}$ reoşã

$$
\left(\mathrm{C}_{2} \mathrm{H}_{5}\right)_{2}{ }^{\mathrm{NH}}+\mathrm{C}_{2} \mathrm{H}_{5} 5^{\mathrm{IH}_{3}}{ }^{+} \rightarrow\left(\mathrm{C}_{2} \mathrm{H}_{5}\right)_{2}{ }^{\mathrm{NH}}{ }^{+}+\mathrm{C}_{2} \mathrm{H}_{5}{ }^{\mathrm{MII}} 2
$$

produz urn $\Delta I$ nogativo. No sentido inverso, obtén-so un $\Delta I$ positivo. 

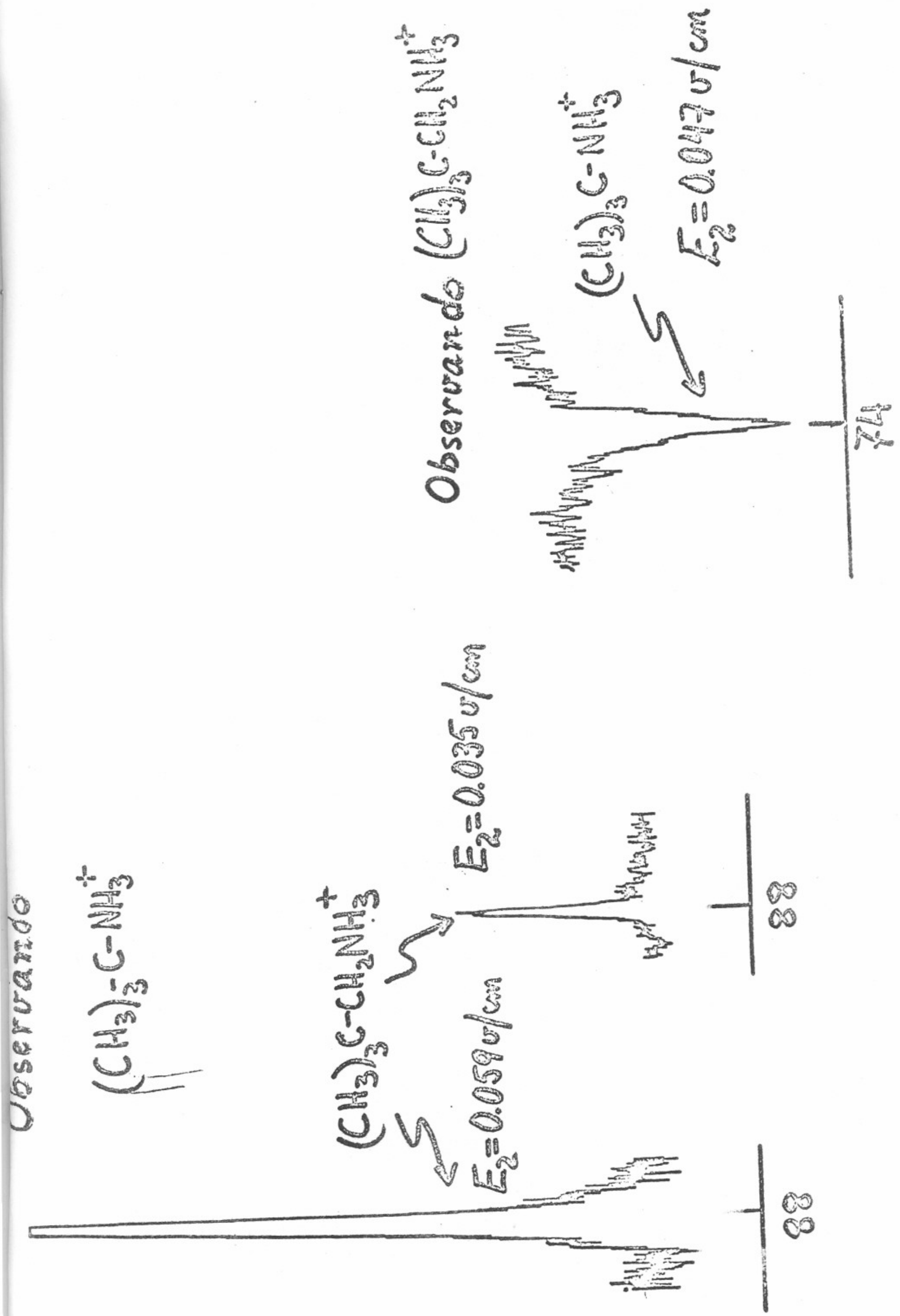

FIGURA

Bspectros de ressonância durla para determinar la basicidade relativa da t-butil mina e a neo-pentil amina. o valor de $\omega_{2}$ se refere a amplitude de rradiofrequência de segundo oscilcdor. 
$\left(\mathrm{C}_{2} \mathrm{H}_{5}\right)_{3}+\left(\mathrm{C}_{2} \mathrm{H}_{5}\right)_{2} \mathrm{HH}$ : Aqui os resultedos concordon com a cor toristica goral que obsorvaume nos casos anteriores. Assing so contrário do que ocorre en fase liquida,

$$
\text { A.P. de }\left(\mathrm{C}_{2} \mathrm{H}_{5}\right)_{3} \mathrm{~N}>\mathrm{A} \cdot \mathrm{P} \cdot \mathrm{de}\left(\mathrm{C}_{2} \mathrm{H}_{5}\right)_{2} \mathrm{NH}
$$

$\left(\mathrm{CH}_{3}\right)_{2} \mathrm{CHNH}_{2}+\left(\mathrm{CH}_{3}\right)_{2} \mathrm{NH}$ : Para esta mistura, as duas roaçõos estudadas, de protonação de dimetil amine por $\left(\mathrm{CH}_{3}\right)_{2} \mathrm{CHNH}_{3}{ }^{+}$ c de protonação da isopropil anina por $\left(\mathrm{CH}_{3}\right)_{2} \mathrm{NH}_{2}{ }^{+}$, produziron un $\Delta I$ nogotivo. Aintorprotação dêsto resultado foi antecipada hos esclarecimentos da parte III.5. A única diferença observada entre as duas reações é o fato de o $\Delta I$ ser bom mais fraco para a prineira reação.

$\left(\mathrm{CH}_{3}\right)_{2} \mathrm{NH}+\mathrm{CH}_{3} \mathrm{CH}_{2} \mathrm{CH}_{2} \mathrm{NH}_{2}$ : Os espectros de ressonência dupla fornecom nêste caso urn rosultado muito interessanto, levando om conte os resultados obtidos no experiêneic anterior.

$$
\text { A.P. de }\left(\mathrm{CH}_{3}\right)_{2} \mathrm{NH}>\text { A.P. de } \mathrm{CH}_{3} \mathrm{CH}_{2} \mathrm{CH}_{2} \mathrm{NH}_{2} \text {. }
$$

$\left(\mathrm{CH}_{3}\right)_{3} \mathrm{~N}+\left(\mathrm{CH}_{3}\right)_{3} \mathrm{CNH}_{2}:$ Aqu1, os resultados foram novanonte de interpretação fRcil, tendo se obtido as caraterlsticas dos reações nos dois sentidos.

$$
\text { A.P. de }\left(\mathrm{CH}_{3}\right)_{3} \mathrm{~N}>\text { A.P. de }\left(\mathrm{CH}_{3}\right)_{3} \mathrm{CNH}_{2}
$$

$\left(\mathrm{CH}_{3}\right)_{3} \mathrm{~N}+\left(\mathrm{C}_{2} \mathrm{H}_{5}\right)_{2} \mathrm{NH}$ : (Ver figure 3-7). As ducs recȩões de protonação estudadas produzen un $\Delta I$ nogetivo, concluindo-so então quo dontro dos Iimites do metodo utilizado,

A.P. de $\left(\mathrm{CH}_{3}\right)_{3} \mathrm{~N} \approx \mathrm{A} . P$. de $\left(\mathrm{C}_{2} \mathrm{H}_{5}\right)_{2} \mathrm{NH}$ 


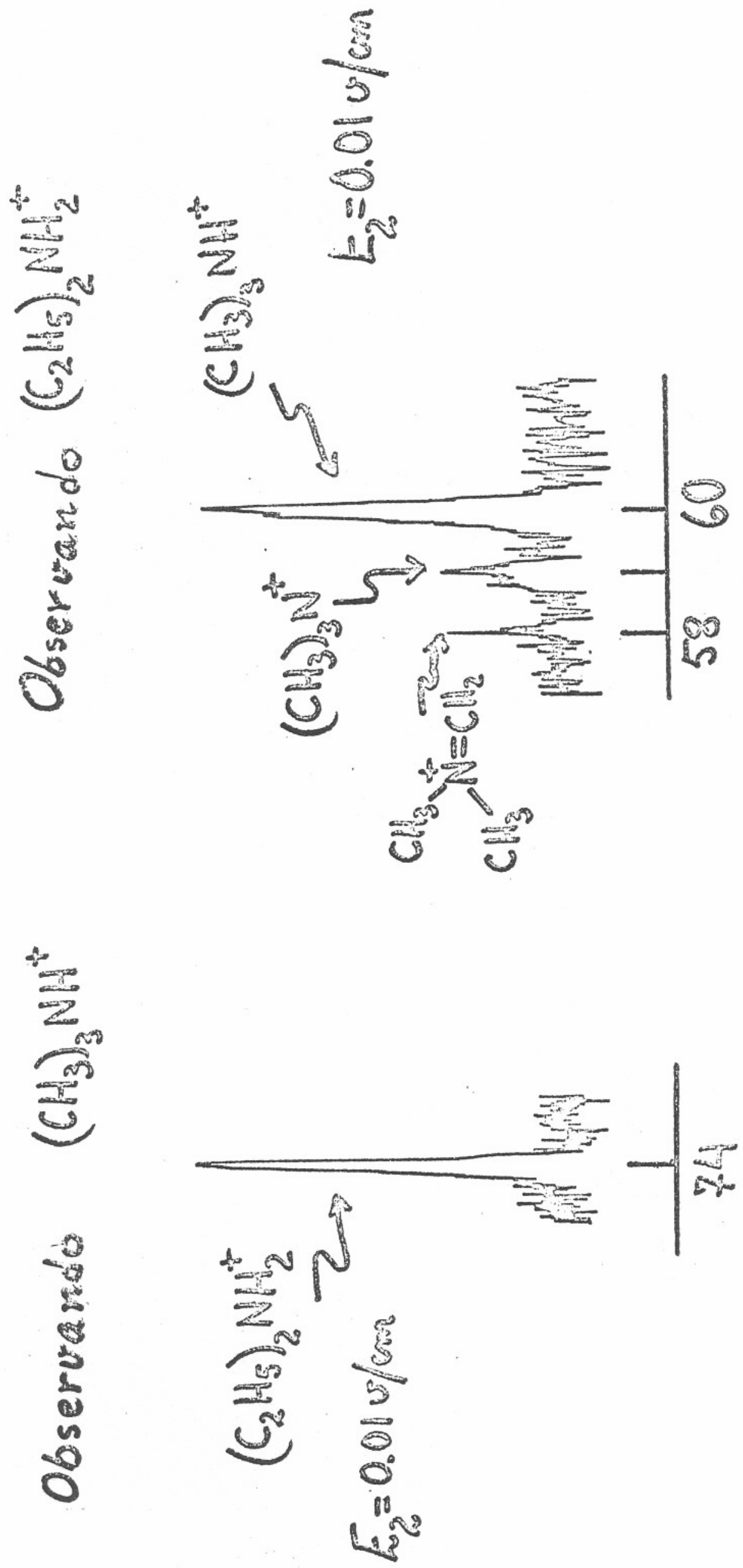

FIGURA $\quad 3-7$

Tspectros de ressoncncia dupla usados pra detemintr a basicidade relativá da trimetil amina e a dietil amina. 
III.7 Anélise critico de or dor de bosicidade por ressonêneie duple

As conclusões obtidas atravós das exporiancias de ressonencia dupla de misturas de onines pomitom construir una escola rolativa de ofinidade protônica, o de basiciade, dos aminas. Porsm, a validoz na interpretação dos rosultados dependo de una sorie de fatores que precisem ser cnalisados separanente cintes que o.s conclusões dêste trobelho sejan postuledes.

a) Superposição no espectro de dois fons de estruturo diferente mas com a mesma massa.

Liste possibilidade pode ser considernda con ajuda do figura 3-2. Nêssc espactró, os lons $\mathrm{CH}_{3} 3^{\mathrm{IHH}}{ }^{+} \mathrm{C}^{\mathrm{C}_{2} \mathrm{H}_{5} 5^{\mathrm{MH}}}{ }^{+}$tôm sido identificados como rosponsávois pelos picos de masse 32

c 46 rospectivemente. E' obvio quo os conclusões cinéticas e tomodinâmicas obtidas da figure 3-3 são vélidas somonte so os picos detectados ne figura 3-2 corrospondon 0.0 fons idontificados.

Batas dúvidas poden sor esclurecidos ctravés dos espectros de rossonêncio simples dos aninas individunis. Do feto, observou-se que une deteminada crina não produzia no ospectro simples un fon de mossa igual deruele fon protonado de outro mine com o qual ostario ligedo na experiência de ressonêneio dupla. Por outro lado, os lons protondos utilizados no rossonencic duplau, de masse. M+I, são corcterísticos dos ospectros do RCI devido is condiçõos ravoréveis pere a observação de fons jocundérios. Assin, os espectros da feferôncio 25 mostron a ousôncio dêste pico nos esjectros de mossc cumuns. 
Ume segunde dificuldade da mosmo noturoze oprosente una situação bom mais sútil. Efotivomente, os lons do messe M+1 têm sido identificados como produtos do protonaç̃o no nitrogênio e não nos carbonos. Hsta conclusão este bascada om crgumentos quínicos intuitivos que ostabolecom o nitrogênio como scndo meis bśsico do que o curbono. Porém, a não contribuição de 1ons protonados no carbono, mesmo que pequene, foi ostabelocida experimentalnente por Blair 41 uscndo otil mine, coutcrada nos étomos ligcdos nos carbonos.

b) Equilfbrio témico nos graus de liberacde internos dos reagentes.

Os dados temodinênicos a serem derivados para as recçõs estudadas, e as suas implicações do ponto de vista físico-químico, visom estcbolccer propriedades fundornentais das oninos. Para consoguir Este objotivo, as grondezas temodinâmicas dovem estar baseadas en roações nas quais as moléculas reagentes, assim como os produtos, estão en scus ostcdos estecionkrios fundomontais. Para deteminações que produzom una grandeze on funç̃o do tomperatura, rocgentos o produtos devon oster caroterizados por ume distribuição estatística do Boltzmann nos grous de liberdade intomos. Estes requisitos são críticos para o coso dos grous de liberdade elotrônicos e vibrocioncis quo podom altercr os resultados de vérios $\mathrm{kcol} / \mathrm{mol}$.

Para julgor a importância dôste detalhe nas implicações tircdes das oxperiôncias de ressonâncio dupla, e necosserio considerar o procosso através do qual os lons sc̃o lomados. Os lons protonados das aminas são fons sccunct́rios, formados a maior perte das vêzes por reaçoos de lons prinérios, ou 
fragmentos de 1ons prinfrios, com os minos noutros. Astos Ions primérios sz̃o fomndos por impecto olotrônico an procossos que do ponto de vista estacionério ostão rogulados por futoros de Franck-Condon correspondentes $c$ duns curvas de potonciol num espeço multidimonsional de 3N-6 graus vibracioncis. Isto implica om que os lons fomados por inpacto eletronico, ou mosmo por ercogmentação, podem tor excitação elotrônica, vibrocional e rotacional.

A posífvel excitação eletrônica não é de conscquêncio imediata para nosso caso, je que a vida dos estados cletrônicos oxcitados é muito curta con relação de froquência média de colis̃̃o reativa. A excitaça rotocional b dosnrozívol pola sua ordon de grandoza. Introtanto, o problomo de excitoç̃̃o vibrccionol é diferente, porque na maioria das vêzes a relaxação desse onerBia deveré scr feite ctrovés de colisões com outras moléculcs. Como a constanto de velociade pera resçoes entre íns e moléculas \& gerolnonte muito alta, existo a possibilidade que íons coir vórios quonta de energie vibracional roogon poro fomar produtos com ume distribuição vibracional dosconhocida. En rosumo, nos condições en que a exporiêncie e roalizade no ospectrômetro de RCI não hé necesshriomonte uns distribuiçño ostotistica de Boltzmann entre os níveis vibracionais dos diferentes fons. As Iinitações que estas condições podon impôr nos conciusões são ressaltodas pola rocento controvérsia ocasionada polo determinação da afinidade protônica de $\mathrm{H}_{2} \mathrm{O}$ por Friedmon ${ }^{42}$ e por Beaucharap e Butrill. 39

As consequências dostos aprecicȩões no nosso trabclho não podom ser avaliadas quantitativanente. Justenento, os procossos de excitação por cletrons, reloxação de cnergio vibrccionel, 
e distribuiçõo vibracional cos produtos do une roaçõo químico constituem una das brocs nois frscincntos da físico-quínice. atual, e só oxistern conhecimentos detclhndos pore os cosos mois simples. Isto possível dificuldndo foi ontõo igmorado neste caso por fulta de dados. Assim, as renços de tranforôncia do protons entro as aminas cqui considerodos prossupõon oquilíbrio témico intomo, e por tonto a inplicução enorgética da expriêncie de ressonêncie duple é admitide como sendo indicative das propriedados intrinscens dos ominos. Lstas hipotosos ñ̃o podem ser justificndas rigoroscrente, mbora existon eois fatores a sou fevor. Do futo, foi monciondo quo os espoctros de ressonêncic duple obtidos a onorgios cletrônicas aiforentos não produziram mudanças no sinal algébrico do $\Delta I$. Isto indico quo a onerģie do projétil ionizonto não voria a distribuiço de onergia interna dos fons estudados com rolação a onorgia interne presonto ne tronsiçõo puromento verticol pemitidu polo fator de Frnnck-Condon. Ilais corretmonto, deverimos rostringir oste ofirmaç̃o cos grous vibrceionais de importincio ne rosç̃o quimica considerada. Ho segundo lugr, as aninas possuon muitos graus de libordade que ajudorion no relowanonto do onergin, vibracional. Por tonto, uno distribuição tómica do Boltzmonn scria obtida mois repicanonte do que poro um sistone triatomico como $\mathrm{H}_{2} \mathrm{O}$.

c) Inforência da bosicidade intrínsece a partir de dodos do cntelpia.

As conclusões obtidas a portir dos experiencins rolotadas na parte III.5 pernitom construir alon de une oscal? relativa de afinidade protônica, une escale de basiciacte. 
De fcto, as roaçôs do tipo (3--15) estĩo acompanhodas do un $\Delta S \neq 0$, cono pode sor percobido qualitotivanonte consideramdo as contribuições estotísticas nas funções de partição de produtos e rocgentes. Dosto manoira, é possívol afimar que o $\Delta H$ parc a roag̃a (3-15) seró igual 00 AG. A semolhonģa estrutural cntre reagontes o produtos pemito tombém estabolecer que as grondezas temodinâmicas deste reaçõo serño rolativononto independentes do tomportura. Por tonto, O $\triangle H$ detominado quelitctivamento nestas experiencias i tomporoturn ambionte são considercdas como correspondente a efeitos intrínsocos dos sistoncs considercdos.

III.8 Hacla de bosicidade relotive das aminos

Os resultados obtidos porn os misturas do aminas pelo mbtodo de ressonêncic dupla podem sor resumidos nume tebele de basicidade relativo on ordon croscente de basicidade.

$$
\begin{aligned}
& \mathrm{NH}_{3}<\mathrm{CH}_{3} \mathrm{NH}_{2}<\mathrm{C}_{2} \mathrm{H}_{5} \mathrm{NH}_{2}<\mathrm{CH}_{3} \mathrm{CH}_{2} \mathrm{CH}_{2} \mathrm{HH}_{2}<\left(\mathrm{CH}_{3}\right)_{2} \mathrm{CHNH}_{2} \\
& \left(\mathrm{CH}_{3}\right)_{2} \mathrm{CHNH}_{2} \simeq\left(\mathrm{CH}_{3}\right)_{2} \mathrm{IH}<\left(\mathrm{CH}_{3}\right)_{3} \mathrm{CHCH}_{2} \mathrm{NH}_{2}<\left(\mathrm{CH}_{3}\right)_{3} \mathrm{ClNH}_{2} \\
& \left(\mathrm{CH}_{3}\right)_{3} \mathrm{C}-\mathrm{NH}_{2}<\left(\mathrm{CH}_{3}\right)_{3} \mathrm{~N} \approx\left(\mathrm{C}_{2} \mathrm{H}_{5}\right)_{2} \mathrm{NH}<\left(\mathrm{C}_{2} \mathrm{HH}_{3}\right)^{\mathrm{N}}
\end{aligned}
$$

Bsta tabcla constitui a conclusão mais importanto dôsto trabalho.

A relação ontre a metil mine o mônia foi obtida da reforência 24 .

A unica arvida que oxisto no tabela se refore c̀ comperaşão entro $\mathrm{CH}_{3} \mathrm{CH}_{2} \mathrm{CH}_{2} \mathrm{NH}_{2}$ e $\left(\mathrm{CH}_{3}\right)_{2} \mathrm{CHIH}_{2}$. Como os Íons protonndos destas duas aninas tôm extomento a mosma massa, não foi possível fazer un estudo direto da roeç̃o de tronsforencis de próton entre elas. Pora indicar que $\left(\mathrm{CH}_{3}\right)_{2} \mathrm{CHNH}_{2}$ ó mais bésico 
ontre as cucs propil minas usou-so critério indiretos o suc interprotoç̃o pode ser questionoda. 0 ecto de $\mathrm{CH}_{3} \mathrm{CH}_{2} \mathrm{CH}_{2} \mathrm{NH}_{2}$ tor se rovelado monos bísice do que $\left(\mathrm{CH}_{3}\right)_{2} \mathrm{HH}$, onquento que que as experiôncias de $\left(\mathrm{CH}_{3}\right)_{2} \mathrm{CHMH}_{2}$ con a diretil anino não pormitom identificar una diforonge on bosicidodo, foi utilizado como provo indirota da rolação sugorida. Un ostudo usondo ume das aninas subotituida com coutério serio una noneira do se ostudar diretenonto esta roleção.

\section{IIJ.9 Consideracõos fineis}

Ne introduço dôste torcoiro capitulo foi resscltadc a importância de se conhocer a bosiciade intrínsece de uno série de compostos orgênicos semelhontes, indepondente des contribuições devido 20 solvente. INo decorrer dos últinos ftons, a utilidade de ressonêncie ciclotrônice de fons, con cuxilio da técnica de rossonêneia dupla, ten sido domonstrodo pora a deteminação da bosicidade relotive dos minss. 0 nétodo om si oncerra grondes possibilidodes para a constuçẽo de extensos tabolas de afinidades protônicos, consiciercndo sobrotudo es condiçõos fuvoráveis que existom na celo do ospectrômetro pare a formação de lons protonodos. o uso cods vez maior desto técnica ó ilustrado on dues rocontos publicacoosos. 43,44

Os resultodos s̃̃o qualitotivos no sontido de que as experiências só permiton decidir se une substêneia ó mais ou menos bésica do que outra. Porén, os dados são coorontos cntre si, e nunca observou-so una situcçóo na qual a orden de basicidade estobelecida nume experioncis, ontreva on contradição com exporioncios foitas con reloşão a uno terceira substância. 
Observa-se como regro geral que a basicidode das minos primérias, sccundórios o torcí́ries crosce sistemeticasento com - tomonho do grupo alquílico introduzido na molócula. o neconismo especifico responsével por esta tondêncie ñ̃o é fécil de aponter on tômos rigorosos, e não é o objetivo dêsto trab Iho cdontrar-se numa ḱred coborte de crgumentos de vilidez purononte Penomonologica. Enbore sejon feites estas ressalvas, observese que a orden de basicidade acomponhe exatamente a ordem sssociada gorolmento om quínice orgênica con o ofeito indutivo de grupos alquilicos. Assin, rossalta nitidomente o comportomento favorável das substêncins con grupos alquilicos substituidos com motila nas posiçõos $\alpha$. Intão, tomos quo $\left(\mathrm{CH}_{3}\right)_{2} \mathrm{CHNH}_{2}$ ć mais bósico do que $\mathrm{CH}_{3} \mathrm{CH}_{2} \mathrm{CH}_{2} \mathrm{HH}_{2}$, o $\left(\mathrm{CH}_{3}\right)_{3} \mathrm{C}-\mathrm{NH}_{2}$ mais bésica do que $\left(\mathrm{CH}_{3}\right)_{3} \mathrm{C}-\mathrm{CH}_{2} \mathrm{NH}_{2}$.

Estas conclusõos parecom simples e logicos ate serom comparadas com os dados obtidos om estudos roclizados om solução. 45-47 A escola relativa dêsses estudos aprosonta umo tendôncia indefinida o incerte, sondo quo olgumas das detominações sofrom sórics dificuldades experimontais. Talvez, cs nosscs conclusões possum ter valor justomento ne interprotaço do oscalo. obtida no fase Ifquida. Porm, \& evidente que algunas das toorias - oxplicacõos quo surgiran pare clucidar os resultados obtidos en solução, o especificmente o modêlo bon conhecido de Brow, 48 nõo so justifican parn o coso dos aminos. 


\section{RESUIO}

Os aspectos matematicos o If́sicos da espectroscopic de ressonêncic ciclotrônica de Lons sño detolhados poro o uso desto técnica no estudo de renções ontre ions e nolbculas noutras ne fase gasosa.

1) A identificoç̃̃o do lon rengento rosponsével pele fomoção de un fon produto ne cole do espectrônetro pode sor estabelecida selotivanente pelo nétodo do ressonência duple.

2) O sinal obtido nos espectros puros de ressonêncie dupla, obti-dos modulando o oscilcdor do rossonencio duplo, esté Intinononte ligodo d ontalpia de resça químico ostudnda. No coso específico dos procossos do tronsferêncio de proton, uno renção exotérmica occsiona un sinal negativo, onqunto que o inverso é observado pare une reação ondotérmica.

3) Estos conceitos aplicedos no estudo de misturas do minas alifaticas pemite astabelecor a basicidade relative intrínseca dêstos compostos. Os rosultados assin obtidos indepondon dos efeitos de solvatação, e concorden surpreendontenente bom com os conceitos de efeito indutivo do grupos alquila loc lizados perto do nitrocennio. 


\section{BIBI IOGRLPIA}

1) I. Scott, ine Physics of Llectricity and Iognetism (John .iloy and Sons, Inc., Now York,U.S.4. 1959) prog.255-258

2) J.D. Jackson, Classical Bloctrodynonics (John Hiloy and Sons, Inc., Now York, U.S.A., 1962) item 12.7, pog. 411-412

3) H. Sommer, H. Thomas e J。Hiple, Phys.Rev。 82,697(1951)

4) R. . Kiser, Introduction to liass Spoctronetry and its Applicetions, (Prentice Holl, Inc, Englowood Cliffs, U.S.A.,1965) p. 72-78

5) D. Vobschall, J.R. Grahom,Jr. O D.P. Malone, Phys.Rev. I3I, 1565(1963)

6) I.R. Anders, J.I. Bocuchanp, R.C. Dunbor, e J.D. Baldeschwieler, J.Chem.thys. 45,1062(1966)

7) J.I. Becuchamp, I.R. Andors o J.D. Baldoschwieler, J.Am.Chom. Soc. $894569(1967)$

8) J.D. Boldeschwieler, Scicnce 159,263(1958)

9) R.V. Pound e .D. Knight, Rev.Sci.Instr.21,219(1950)

10) G.D. ictkins e R.V. Pound, Phys.Rev. 82,343(1.951)

11) E.R. Androw, Nuclecr Niognetic Resonnce, (Cabridge Univorsity Pross, Combridge, Inglatorr, 1958) pog. 49-53

12) C.P. Poole, Electron Spin Resononce (Intorscionce Publishors,

New York,U.S.L., 1967) pag.452-456

13) J.M.S. Henis e $/$. Frasuro, Rev.Sci.Instr. 39,1772(1968)

14) R.I. MeIver (rosultacios inéditos) (Univorsidade de Stonford, 1969)

15) Referêncic, 12, pas。 394-421

16) I.R. Lnders, tese de Ph.D., Harvord Univorsity (1966)

17) J.I. Boauchamp, toso do Ph.D., Horvard Univorsity (1967)

18) J.I. Boauchamp c J.T. Armstrong, Rov.Sci.Instr.40, 123(1969)

19) M.I. Bowers, D.D. Blleman o J.King,Jro, J.Chon.Phys. 50,4787 (1969)

20) Reforência 2, 1tern 12.8

21) D.C. Kolly, H. Margonau e S.C. Brom, Phys.Rev. 108, 1367(1957)

22) J.I. Bcouchomp, J.Chom.Phys. 46,1231 (1967)

23) J.I. Brauman ॰ I.K. Bloir, J.Lm。Chom.Soc.90,5636(I968)

24) J.I. Braunan o I.K. JIair, J.fm.Chom.Soc. 90,656I( 1968 )

25) R.P. Boll, The Proton in Chomistry (Comell Univorsity Press, Ithaca,U.S.A.) (1959)

26) HoM. Amott, Progross in Physical Organic Chomistry 1,223(1963) (Interscionce Publishors, Now Mork, U.S.A.) 
27) Handbook of Chomistry nd Physics, (The Chemical Rubber Co., 47 th Lition, Clovelond, U.S.A.,1966) peg. D-85

28) M.S.D. Munson, J.Am.Chem.Soc. 87,2332(1965)

29) R.S. Gohlke e F.h. Mc Iafferty, Anal.Chem. 34,1281 (1962)

30) J. Shomon, Chem.Revs. II, 164(1932)

31) V.I. TaI'roze o A.K. Iyubimova, DokI. Lkod.Mouk.SSSR 86,909(1952)

32) D.P. Stovenson e D.O. Schissler, J.Chon.Phys. 29,282(1958)

33) F.H. Fiold, J.I. Frenlelin e F.t. Lampe, J.Ln.Chon.Soc. 79, 24.19 (1957)

34) F. . Iompe, J.I. Franklin o F.H. Ficld, Prog.Reaction Kin. I, 69(I96I) (Pergninon Pross, Now York, U.S.A.)

35) G. Giounousis C D.P. Stovenson, J.Chen.Phys. 29, 294(1958)

36) Do.t. Nic Doniol, Collision Phononone in Ionizod Goses (John

iliey and Sons, Inc., Now York, U.S.L., 1964) pog. 67-75

37) H.S. Johnston, Ges Phase Reaction Rate Theory (The Ronald Pross

Compeny, Now York, U.S.A.,1966) Copítulo 9

38) Advancos in Chonistry Sorios $\mathrm{N}^{\circ}$ 58: "Ion Roleculo Roactions in

the Gos Phase" (Mnorican Chomical Society, (oshington, U.S.L., 1966)

39) J.I. Beauchamp o S.E. Butrill,Jr., J.Chom.Phys.48,1783(I968)

40) J.C. Iight, J.Chom.Phys. 40,3221 (1964)

41) I.K. Blair (trabalho jnédito, Univorsidade de Strnford, 1968)

42) Ni. Do Pas, J.J. Ievonthol e I. Friodmen, J.Chom.Phys. 49,5543 (1968)

43) J.I. Brawnon o I.K. BIair, J.Am.Chon.Soc. 9I,2127(1969)

44) D. Holtz e J.I. Becuchamp, J.Lm.Chom.Soc。 91,5913( 1969)

45) D.D. Perrin, Dissociation Constonts of Organic Dases in heuoous Solution, Buttorworths, Iondon, Inglaterra, 1965)

46) H.K. HaII,Jr. J.Phys.Chom。60,63(1956)

47) L.G. Evans e S.D. Hamann, Trans.Far.Soc.47,34(1951)

48) H.C. Brow, H. Bartholoney,Jr。 O M.D. laylor, J.Am.Chem. Yoc. $66,435(1944)$ 